\title{
Optimal dividend payments for a two-dimensional insurance risk process
}

\author{
Pablo Azcue $^{1} \cdot$ Nora Muler $^{1} \cdot$ Zbigniew Palmowski $^{2}$
}

Received: 26 April 2018 / Revised: 4 September 2018 / Accepted: 2 October 2018 /

Published online: 24 October 2018

(C) The Author(s) 2018

\section{Abstract}

We consider a two-dimensional optimal dividend problem in the context of two branches of an insurance company with compound Poisson surplus processes dividing claims and premiums in some specified proportions. We solve the stochastic control problem of maximizing expected cumulative discounted dividend payments (among all admissible dividend strategies) until ruin of at least one branch. We prove that the value function is the smallest viscosity supersolution of the respective Hamilton-Jacobi-Bellman equation and we describe the optimal strategy. We analyze some numerical examples.

\section{Introduction}

In collective risk theory the surplus process $X$ of an insurance company is modeled as

$$
X(t)=x+c t-S(t),
$$

where $x>0$ denotes the initial surplus,

$$
S(t)=\sum_{i=1}^{N_{t}} U_{i}
$$

is a compound Poisson. We assume that $U_{i},(i=1,2, \ldots)$ are i.i.d. distributed claims (with the distribution function $G$ ). The arrival process is a homogeneous Poisson

Zbigniew Palmowski

zbigniew.palmowski@gmail.com

1 Departamento de Matematicas, Universidad Torcuato Di Tella, Av. Figueroa Alcorta 7350, C1428BIJ Buenos Aires, Argentina

2 Faculty of Pure and Applied Mathematics, Wrocław University of Science and Technology, Wyb. Wyspiańskiego 27, 50-370 Wrocław, Poland 
process $N_{t}$ with intensity $\lambda$. The premium income is modeled by a constant premium density $c$ and often assumed net profit condition $c>\lambda E\left[U_{1}\right]$ gives the unrealistic property that process $X(t)$ converges to infinity. In answer to this objection De Finetti [21] introduced the dividend barrier model for one-dimensional model (1), in which all surpluses above a given level are transferred to a beneficiary. Further, usually the payment of dividends should be made in such a way as to maximize the expected discounted sum of dividends paid up to ruin time. In 1969, Gerber [23] showed that if the free surplus of an insurance portfolio is modelled by a compound Poisson risk model, it is always optimal to pay dividends according to a so-called band strategy, which collapses to a barrier strategy for exponentially distributed claim amounts. Later, lots of works in the mathematical finance and actuarial literature concern the dividend barrier models and the problem of finding an optimal policy for paying out dividends. Gerber and Shiu [22], Grandits et al. [26] and Jeanblanc and Shiryaev [30] considered the optimal dividend problem in a Brownian setting. Irbäck [28] and Zhou [41], Zajic [40], Avram et al. [4], Kyprianou and Palmowski [31], Loeffen [32] studied the constant barrier model for a classical and spectrally negative Lévy risk process. Azcue and Muler [8] follow a viscosity approach to investigate optimal reinsurance and dividend policies in the Cramér-Lundberg model. The most general criteria currently available for barrier strategies to be optimal can be found in Loeffen and Renaud [33].

A detailed overviews on this subject from different perspectives are given in Azcue and Muler [10], Schmidli [36], Albrecher and Thonhauser [2] and Avanzi [5].

All these control problems have been formulated and studied in the one-dimensional framework. However, in recent years there has been an increased interest in risk theory in considering the multidimensional surplus model where $X(t), x, c$ and $S(t)$ are vectors, with possible dependence between the components. Indeed, the assumption of independence of risks may easily fail, for example in the case of reinsurance, when incoming claims have an impact on both insuring companies at the same time. In general, one can also consider situations where each claim event might induce more than one type of claim in an umbrella policy (see Sundt [39]). For some recent papers considering dependent risks, see Dhaene and Goovaerts [19, 20], Goovaerts and Dhaene [25], Müller [34, 35], Denuit et al. [17], Ambagaspitiya [3], Dhaene and Denuit [18], $\mathrm{Hu}$ and $\mathrm{Wu}$ [27] and Chan et al. [14]. Ruin probability expressions for a two-dimensional risk process were also studied in Avram et al. $[6,7]$ for simultaneous claim arrivals and proportional claim sizes and recently in Badila et al. [13] and Ivanovs and Boxma [29] in a more general framework.

In this paper we analyze the dividend problem for the two-dimensional risk model in which two branches of a company with initial capiotals $x_{1}$ and $x_{2}$ split the amount they pay out of each claim in fixed proportions $b_{1}$ and $b_{2}\left(b_{1}+b_{2}=1\right)$, and receive premiums at rates $c_{1}$ and $c_{2}$, respectively. Moreover, these two branches have the same shareholders and the objective is to maximize the total dividends received by these shareholders. That is, the main goal of this paper is identification of the value function maximizing the weighted sum of expected discounted dividend payments until ruin of at least one branch. This will lead to a fully two-dimensional stochastic control problem and we answer the question what the analogues of the optimal univariate barrier strategies are in two dimensions. 
In Azcue and Muler [9], the problem of optimally transferring capital between two portfolios in the presence of transaction costs was considered, see also Badescu et al. [12]. Albrecher et al. [1] considered the problem of optimizing dividends for two collaborating insurance companies whose surpluses are modelled with independent compound Poisson processes.

Czarna and Palmowski [16] studied the same dividend problem as in this paper but for a very particular dividend strategy of reflecting two-dimensional risk process from the line. Solving certain partial differential equations they managed to identify the value function for the exponential claim sizes. We will show in this paper though that this strategy is not optimal.

In this paper we prove that the value function is a viscosity solution of the respective Hamilton-Jacobi-Bellman equation (abbreviated lately by HJB) and it can be characterized as the smallest viscosity supersolution. Using this result we manage to identify the optimal strategy. In particular, under assumption that the second branch receives less premium per amount paid out, for initial surpluses $\left(x_{1}, x_{2}\right)$ satisfying $x_{1} / b_{1}>x_{2} / b_{2}$ the best strategy is to pay $\left(x_{1}-\left(b_{1} / b_{2}\right) x_{2}\right)$ by the first branch immediately as dividends.

For the symmetric case $c_{2} / c_{1}=b_{2} / b_{1}$ and for $x_{1} / b_{1} \leq x_{2} / b_{2}$, by transferring the optimization problem into one-dimensional optimization problem, we managed to describe the optimal strategy explicitly. For $x_{1} / b_{1}<x_{2} / b_{2}$ the optimal strategy is that the second branch immediately pays $x_{2}-\left(b_{2} / b_{1}\right) x_{1}$ as dividends and afterwards the controlled process remains in the half line $\mathcal{M}$ in $\mathbf{R}_{+}^{2}$ that contains the origin with slope $b_{2} / b_{1}$ until ruin time. In $\mathcal{M}$, either both branches pay the incoming premium as dividends until next claim, or the second branch pays a positive amount $m$ of money and the first branch pays $\left(b_{1} / b_{2}\right) m$, where $m$ is the minimal amount that brings the surplus process back to the previous case, or, finally, both branches pay no dividends.

For general, non-symmetric case $c_{2} / c_{1}<b_{2} / b_{1}$ we use a convergent numerical scheme to find the optimal strategy for initial surpluses $\left(x_{1}, x_{2}\right)$ satisfying $x_{1} / b_{1} \leq x_{2} / b_{2}$. More formally, given any $\delta>0$, we denote $\Delta x_{1}=c_{1} \delta$ and $\Delta x_{2}=c_{2} \delta$ and define the grid domain $\mathcal{G}^{\delta}$ in $\mathbf{R}_{+}^{2}$ as $\mathcal{G}^{\delta}:=\left\{\left(n \Delta x_{1}, m \Delta x_{2}\right): n, m \geq 0\right\}$. We look, at each point of the grid $\mathcal{G}^{\delta}$, for the best local strategy among the ones suggested by the operators of the HJB equation. These possible local strategies are: the first branch pays a lump sum as dividends, the second one pays a lump sum as dividends, or none of the branches pay dividends. These local strategies are modified in such a way that the controlled surplus after applying these local strategies lies in the grid. We believe that this techniques used in this paper could be applicable to other risk-sharing mechanisms.

The rest of the paper is organized as follows. In Sect 2 we present the model we deal with and state formally the problem that we want to solve. In Sect. 3 we prove that the value function is a viscosity solution of HJB. Using this result we describe the optimal strategy in some cases in Sect. 4, in Sect. 5 we describe a numerical scheme to approximate the optimal value function and the optimal strategy and in Sect. 6 we present some numerical examples. We conclude the paper with the number of comments in Sect. 7. 


\section{Model}

In this paper we consider two branches of a company that receive premiums at different rates and then split the amount they pay in fixed proportions for each claim. This model corresponds to proportional reinsurance dependence. The total amount of claims up to time $t$ is modeled as a compound Poisson process. We can write this two-dimensional risk model as

$$
\bar{X}(t)=\left(X_{1}(t), X_{2}(t)\right)=\left(x_{1}+c_{1} t-\sum_{i=1}^{N_{t}} b_{1} U_{i}, x_{2}+c_{2} t-\sum_{i=1}^{N_{t}} b_{2} U_{i}\right) .
$$

Above $x_{1}$ and $x_{2}$ are the corresponding initial surplus levels and $c_{1}$ and $c_{2}$ are the corresponding premium rates. The sizes of the claims $U_{i}$ are non-negative i.i.d. random variables with absolutely continuous distribution $G$. The claims arrival process $N_{t}$ is a Poisson processes with intensity $\lambda$. Finally, the constants $b_{1}$ and $b_{2}$ are the proportions of the claim that each branch pays, so $b_{1}+b_{2}=1, b_{1}>0$ and $b_{2}>0$. We assume here that the process $N_{t}$ and the random variables $U_{i}$ are independent of each other. Without loss of generality, we can assume that the second branch receives less premium per amount paid out, that is

$$
c_{1} / b_{1} \geq c_{2} / b_{2} \text {. }
$$

We denote by $\left\{\mathcal{F}_{t}\right\}_{\{t \geq 0\}}$ the right-continuous natural filtration of $\bar{X}(t)$ satisfying usual conditions. Throughout this paper all stopping times and martingales are taken with respect of this filtration.

Both branches use part of their surpluses to pay dividends. The dividend payment strategy $\bar{L}(t)=\left(L_{1}(t), L_{2}(t)\right)$ is the total amount of dividends paid by the two branches up to time $t$. We define the associated controlled process with initial surplus $\left(x_{1}, x_{2}\right)$ as

$$
\bar{X}^{\bar{L}}(t)=\bar{X}(t)-\bar{L}(t) .
$$

The dividend payment strategy $\bar{L}=\left(L_{1}(t), L_{2}(t)\right)_{t<\tau}$ is called admissible if it is non-decreasing, càglàd (left continuous with right limits), predictable with respect to the filtration generated by the bivariate process $\bar{X}(t)$ and satisfies $L_{1}(t) \leq X_{1}(t)$, $L_{2}(t) \leq X_{2}(t)$. The first time when the two-dimensional risk process first leaves the positive quadrant will be our ruin time:

$$
\hat{\tau}=\inf \left\{t: X_{1}(t)<0 \text { or } X_{2}(t)<0\right\} .
$$

That is, the ruin time is the first time at which at least one branch get ruined. We denote by $\mathbf{R}_{+}=[0, \infty)$ and by $\mathbf{R}_{+}^{2}=(0, \infty)^{2}$ the first quadrant. Let $\Pi_{x_{1}, x_{2}}$ be the set of admissible dividend strategies with the initial surplus $\bar{x}=\left(x_{1}, x_{2}\right) \in \mathbf{R}_{+}^{2}$. Given an 
admissible dividend strategy $\bar{L} \in \Pi_{x_{1}, x_{2}}$, the expected discounted dividends paid by the two branches until $\widehat{\tau}$ is

$$
V_{\bar{L}}(\bar{x})=E_{\bar{x}}\left(\int_{0}^{\hat{\tau}} e^{-q s} d L_{1}(s)+\int_{0}^{\hat{\tau}} e^{-q s} d L_{2}(s)\right)
$$

where $q>0$ is a constant discount factor.

The main goal of this paper is identification of the optimal value function defined by:

$$
V(\bar{x})=\sup _{\bar{L}_{\in} \Pi_{\bar{x}}} V_{\bar{L}}(\bar{x})
$$

for $\bar{x} \in \mathbf{R}_{+}^{2}$.

In the next section, we will see that $V$ is well defined. Another crucial problem that we treated in this paper is the existence of an optimal strategy defined as follows. Given a family of admissible strategies

$$
\pi=\left\{\bar{L}_{\bar{x}} \in \Pi_{\bar{x}} \text { for each } \bar{x} \in \mathbf{R}_{+}^{2}\right\}
$$

we define the value function $V_{\pi}: \mathbf{R}_{+}^{2} \rightarrow \mathbf{R}_{+}$as $V_{\pi}(\bar{x})=V_{\bar{L}_{\bar{x}}}(\bar{x})$. We say that $\pi^{*}$ is the optimal strategy if $V_{\pi^{*}}=V$.

Remark 1 We can also consider the possibility that the two branches of the company can merge with a merger cost $m$ (see e.g. Gerber and Shiu [24]). The merged company has initial surplus $x_{1}+x_{2}-m$, receives the premium rate $c_{1}+c_{2}$ and pays the whole claims $U_{i}$. The new company uses part of the surplus to pay dividends to the shareholders up to the time in which the joined surplus becomes negative. In this case, the uncontrolled surplus process is given by

$$
X_{M}(t)=x_{1}+x_{2}-m+\left(c_{1}+c_{2}\right) t-\sum_{i=1}^{N_{t}} U_{i},
$$

and the controlled surplus process is given by $X_{M}^{L}(t)=X_{M}(t)-L(t)$, where the dividend payment strategy $L(t)$ is non-decreasing, càglàd (left continuous with right limits), predictable with respect to the filtration generated by the process $X_{M}(t)$ and satisfies $L(t) \leq X_{M}(t)$. We consider the ruin time of the merged company $\tau_{M}^{L}=\inf \left\{t: X_{M}^{L}(t)<0\right\}$ and we define the merger optimal value function as 


$$
V_{M}\left(x_{1}+x_{2}-m\right)=\sup _{L} E_{\bar{x}}\left(\int_{0}^{\tau_{M}^{L}} e^{-q s} d L(s)\right)
$$

for $x_{1}+x_{2} \geq m$. The function $V_{M}$ is the optimal value function of the one-dimensional De Finetti's problem corresponding to the compound Poisson process $X_{M}$.

Let us assume that $m=0$, then given any $\bar{x} \in R_{+}^{2}$ and any admissible strategy $\bar{L}(t)=\left(L_{1}(t), L_{2}(t)\right) \in \Pi_{x_{1}, x_{2}}$, we consider the one-dimensional payment strategy

$L(t)=L_{1}(t)+L_{2}(t)$ for the merged company. Since $\tau_{M}^{L} \geq \hat{\tau}$ we conclude that $V_{M}\left(x_{1}+x_{2}\right) \geq V(\bar{x})$. Note that this is not longer true as $m>0$.

\section{Properties of the optimal value function}

In this section we first state some basic results concerning regularity and growth at infinity of the optimal value function $V$ defined in (6). We then deduce the Hamilton-Jacobi-Bellman equation associated to this optimization problem and see that $V$ is a viscosity solution of the HJB equation. Moreover, we can characterize $V$ as the smallest viscosity supersolution of this equation with a suitable growth condition. Finally, we obtain a verification result: any viscosity supersolution which is a value function of a family of admissible strategies is the optimal value function.

The following two lemmas are the two-dimensional counterparts of Lemmas 2.1 and 2.2 of [8].

Lemma 3.1 For all $\left(x_{1}, x_{2}\right) \in \mathbf{R}_{+}^{2}$ the optimal value function is well defined and satisfies

$$
x_{1}+x_{2}+\frac{c_{1}+c_{2}}{q+\lambda} \leq V\left(x_{1}, x_{2}\right) \leq x_{1}+x_{2}+\frac{c_{1}+c_{2}}{q} \text {. }
$$

Proof Taking strategy that pays at the beginning the whole surpluses $x_{1}+x_{2}$ and then paying incoming premium as dividends up to the first claim arrival $\tau_{1}$ gives the lower bound since

$$
V\left(x_{1}, x_{2}\right) \geq x_{1}+x_{2}+\left(c_{1}+c_{2}\right) E \int_{0}^{\tau_{1}} e^{-q t} d t=x_{1}+x_{2}+\frac{c_{1}+c_{2}}{q+\lambda} .
$$

Similarly, observation that $L_{i}(s) \leq x_{i}+c_{i} s(i=1,2)$ produces the upper bound.

Lemma 3.2 The optimal value function $V$ is increasing, locally Lipschitz and satisfies 


$$
h \leq V\left(x_{1}+h, x_{2}\right)-V\left(x_{1}, x_{2}\right) \leq\left(e^{(q+\lambda) h / c_{1}}-1\right) V\left(x_{1}, x_{2}\right)
$$

and

$$
h \leq V\left(x_{1}, x_{2}+h\right)-V\left(x_{1}, x_{2}\right) \leq\left(e^{(q+\lambda) h / c_{2}}-1\right) V\left(x_{1}, x_{2}\right)
$$

for any $\left(x_{1}, x_{2}\right) \in \mathbf{R}_{+}^{2}$ and any $h>0$.

Proof To prove the lower inequality in the first series of inequalities, it suffices to consider the strategy that pays $h$ as dividends from the surplus of the first branch and then follows the strategy $\pi_{\epsilon}$ such that $V_{\pi_{\epsilon}}\left(x_{1}, x_{2}\right) \geq V\left(x_{1}, x_{2}\right)-\epsilon$ for general $\epsilon>0$. To prove the upper inequality, it suffices to consider the strategy that pays no dividends up the first passage time of $x_{1}+h$ by the first surplus process $X_{1}(t)$ and the follows strategy $\pi_{\epsilon}$ such that $V_{\pi_{\epsilon}}\left(x_{1}+h, x_{2}\right) \geq V\left(x_{1}+h, x_{2}\right)-\epsilon$ for general $\epsilon>0$. The second line of inequalities could be proved in a similar way. The proof of the fact that $V$ is increasing and locally Lipschitz follows now classical arguments.

In order to obtain the Hamilton-Jacobi-Bellman (HJB) equation associated to the optimization problem (6), we need to state the so-called Dynamic Programming Principle (DPP). Since $\bar{X}(t)$ is a Markov process the proof follows the same arguments as the ones given in Lemma 1.2 of Azcue and Muler [10]. They use only the fact that $V$ is increasing and continuous in $\mathbf{R}_{+}^{2}$. Obviously we need also to extend the definition of $V$ to $\mathbf{R}^{2}$ defining $V$ as zero outside the first quadrant.

Lemma 3.3 For any initial surplus $\bar{x}$ in $\mathbf{R}_{+}^{2}$ and any stopping time $\tau$, we have

$$
V(\bar{x})=\sup _{\bar{L} \in \Pi_{\bar{x}}} E_{\bar{x}}\left(\int_{0}^{\tau \wedge \hat{\tau}} e^{-q s} d L_{1}(s)+\int_{0}^{\tau \wedge \hat{\tau}} e^{-q s} d L_{2}(s)+e^{-q(\tau \wedge \hat{\tau})} V\left(X_{1}^{\bar{L}}(\tau \wedge \hat{\tau}), X_{2}^{\bar{L}}(\tau \wedge \hat{\tau})\right)\right) .
$$

We now deduce the HJB equation assuming some regularity on $V$.

For any continuously differentiable function $\mu$ defined in $\mathbf{R}_{+}^{2}$, we define the infinitesimal generator $\widetilde{\mathcal{G}}$ of the controlled process $\bar{X}(t \wedge \hat{\tau})$ by

$$
\widetilde{\mathcal{G}} u(\bar{x}):=\lim _{t \rightarrow 0} \frac{E_{\bar{x}}\left(e^{-q t} u\left(\bar{X}^{\bar{L}}(t \wedge \hat{\tau})\right)-u(\bar{x})\right)}{t}
$$

see $[10$, Sec. 1.4$]$ for details.

We will consider now the admissible strategy $\bar{L}$ in which both branches pay dividends with constant rates $l_{1} \geq 0$ and $l_{2} \geq 0$ respectively until ruin time $\hat{\tau}$. Then using [10, Rem. 1.8] we have

$$
\begin{aligned}
\tilde{\mathcal{G}} u(\bar{x})= & \left(c_{1}-l_{1}\right) u_{x_{1}}(\bar{x})+\left(c_{2}-l_{2}\right) u_{x_{2}}(\bar{x}) \\
& -(q+\lambda) u(\bar{x})+\mathcal{I}(u)(\bar{x}),
\end{aligned}
$$


where

$$
\mathcal{I}(u)(\bar{x})=\lambda \int_{0}^{\left(x_{1} / b_{1}\right) \wedge\left(x_{2} / b_{2}\right)} u\left(x_{1}-b_{1} \alpha, x_{2}-b_{2} \alpha\right) d G(\alpha)
$$

and $\tau_{1}$ denotes the first claim arrival.

Assume now that $V$ is continuously differentiable. Note that from Lemma 3.3 we have:

$$
V(\bar{x}) \geq\left(l_{1}+l_{2}\right) E_{\bar{x}} \int_{0}^{\tau_{1}} e^{-q t} d t+E_{\bar{x}} e^{-q\left(t \wedge \tau_{1}\right)} V\left(\bar{X}^{\bar{L}}\left(t \wedge \tau_{1}\right)\right)
$$

Thus by (8) dividing above inequality by $t$ and taking $t \downarrow 0$ give:

$$
\mathcal{L}(V)(\bar{x})+l_{1}\left(1-V_{x_{1}}(\bar{x})\right)+l_{2}\left(1-V_{x_{2}}(\bar{x})\right) \leq 0,
$$

where

$$
\mathcal{L}(V)(\bar{x}):=c_{1} V_{x_{1}}(\bar{x})+c_{2} V_{x_{2}}(\bar{x})-(q+\lambda) V(\bar{x})+\mathcal{I}(V)(\bar{x}) .
$$

Taking $l_{1}=l_{2}=0, l_{1} \rightarrow \infty$ with $l_{2}=0$; and $l_{2} \rightarrow \infty$ with $l_{1}=0$, we obtain

$$
\max \left\{\mathcal{L}(V)(\bar{x}), 1-V_{x_{1}}(\bar{x}), 1-V_{x_{2}}(\bar{x})\right\} \leq 0 .
$$

We now associate to our problem the following HJB equation:

$$
\max \left\{\mathcal{L}(V), 1-V_{x_{1}}, 1-V_{x_{2}}\right\}=0 \text {. }
$$

Since the optimal value function could be not differentiable, we have to use the notion of viscosity solution and see that $V$ is a viscosity solution of the associated HJB equation. Let us define this notion (see for instance Crandall and Lions [15] and Soner [37]).

Definition 3.4 A locally Lipschitz function $\bar{u}: \mathbf{R}_{+}^{2} \rightarrow \mathbf{R}$ is a viscosity supersolution of (12) at $\bar{x} \in \mathbf{R}_{+}^{2}$ if any continuously differentiable function $\varphi: \mathbf{R}_{+}^{2} \rightarrow \mathbf{R}$ with $\varphi(\bar{x})=\bar{u}(\bar{x})$ such that $\bar{u}-\varphi$ reaches the minimum at $\bar{x}$ satisfies

$$
\max \left\{\mathcal{L}(\varphi)(\bar{x}), 1-\varphi_{x}(\bar{x}), 1-\varphi_{x_{2}}(\bar{x})\right\} \leq 0
$$

A function $\underline{u}: \mathbf{R}_{+}^{2} \rightarrow \mathbf{R}$ is a viscosity subsolution of (12) at $\bar{x} \in \mathbf{R}_{+}^{2}$ if any continuously differentiable function $\psi: \mathbf{R}_{+}^{2} \rightarrow \mathbf{R}$ with $\psi(\bar{x})=\underline{u}(\bar{x})$ such that $\underline{u}-\psi$ reaches the maximum at $\bar{x}$ satisfies

$$
\max \left\{\mathcal{L}(\psi)(\bar{x}), 1-\psi_{x_{1}}(\bar{x}), 1-\psi_{x_{2}}(\bar{x})\right\} \geq 0
$$


A function $u: \mathbf{R}_{+}^{2} \rightarrow \mathbf{R}$ which is both a supersolution and subsolution at $\bar{x} \in \mathbf{R}_{+}^{2}$ is called a viscosity solution of (12) at $\bar{x} \in \mathbf{R}_{+}^{2}$.

Theorem 3.5 V is a viscosity solution of the HJB equation (12) at any $\bar{x} \in \mathbf{R}_{+}^{2}$.

Proof The proof that $V$ is a viscosity supersolution is similar to the one in Proposition 3.1 in [10]. We underline only crucial adjustments that should be made in the proof.

The proof that $V$ is a viscosity supersolution follows the same arguments as the ones used to derive (11).

The proof of the fact that $V$ is a viscosity subsolution is done by contradiction. We will use the following convention. For any vectors $\bar{a}=\left(a_{1}, a_{2}\right)$ and $\bar{b}=\left(b_{1}, b_{2}\right)$ we denote $[\bar{a}, \bar{b}]:=\left[a_{1}, b_{1}\right] \times\left[a_{2}, b_{2}\right]$ with $[-\infty, \bar{b}]:=\left[-\infty, b_{1}\right] \times\left[-\infty, b_{2}\right]$ and for any $h>0$ we will write $\bar{a} \pm h:=\left(a_{1} \pm h, a_{2} \pm h\right)$. We assume that for some fixed $x_{0}=\left(x_{01}, x_{02}\right)$ there exist $\epsilon>0$ and $h \in\left(0, \frac{1}{2} x_{01} \wedge x_{02}\right)$ and test function $\psi$ such that:

$$
1-\psi_{x_{i}}(\bar{x}) \leq 0, \quad i=1,2
$$

for $\bar{x} \in\left[0, \bar{x}_{0}+h\right]$,

$$
\mathcal{L}(\psi)(\bar{x}) \leq-2 q \epsilon
$$

for $\bar{x} \in\left[\bar{x}_{0}-h, \bar{x}_{0}+h\right]$,

$$
V(\bar{x}) \leq \psi(\bar{x})-2 \epsilon
$$

for $\bar{x} \in\left[-\infty, \bar{x}_{0}-h / 2\right] \cup\left\{\bar{x}_{0}+h\right\}$. Now the proof will go along the lines of Proposition 3.1 in [10] by taking $\left(x_{1}-x_{01}\right)^{2}\left(x_{2}-x_{02}\right)^{2}$ and $x_{01}^{2} x_{02}^{2}$ instead of $\left(x-x_{0}\right)^{2}$ and $x_{0}^{2}$, respectively. One needs to modify also the definitions $\frac{2}{\tau}$ and $\underline{\tau}$ into the following ones:

$$
\bar{\tau}=\inf \left\{t \geq 0: X_{1}(t) \geq x_{01}+h \quad \text { or } \quad X_{2}(t) \geq x_{02}+h\right\}
$$

and

$$
\underline{\tau}=\inf \left\{t \geq 0: X_{1}(t) \leq x_{01}-h \quad \text { or } \quad X_{2}(t) \leq x_{02}-h\right\} .
$$

In the last step (see inequality (3.21) in [10]) we use the following zero expectation martingale

$$
\begin{aligned}
\tilde{M}_{\psi}(t)= & \sum_{\substack{X_{1}\left(s^{-}\right) \neq X_{1}(s) \\
s \leq t}}\left(\psi(\bar{X}(s))-\psi\left(\bar{X}\left(s^{-}\right)\right) e^{-q s}\right. \\
& -\lambda \int_{0}^{t} e^{-q s} \int_{0}^{\frac{X_{1}\left(s^{-}\right)}{b_{1}} \wedge \frac{X_{2}\left(s^{-}\right)}{b_{2}}}\left(\psi\left(\bar{X}\left(s^{-}\right)-\alpha\left(b_{1}, b_{2}\right)\right)-\psi\left(\bar{X}\left(s^{-}\right)\right)\right) d G(\alpha) d s,
\end{aligned}
$$


that could be defined properly by Dynkin's formula for any test function for viscosity subsolution $\psi$; see also [10, Prop. 2.13]. At the end after some manipulations we derive that

$$
V\left(\bar{x}_{0}\right) \leq \psi\left(\bar{x}_{0}\right)-\epsilon
$$

which is a contradiction with the assumption that $V\left(\bar{x}_{0}\right)=\psi\left(\bar{x}_{0}\right)$. This completes the proof.

Remark 2 The functions $U\left(x_{1}, x_{2}\right)=x_{1}+x_{2}+K$ are viscosity solutions of the HJB equation (12) for $K>\left(c_{1}+c_{2}\right) / q$ because

$$
\begin{aligned}
\mathcal{L}(U)(\bar{x}) & =c_{1}+c_{2}-(q+\lambda)\left(x_{1}+x_{2}+K\right)+\lambda \int_{0}^{\left(x_{1} / b_{1}\right) \wedge\left(x_{2} / b_{2}\right)}\left(x_{1}+x_{2}-\alpha+K\right) d G(\alpha) \\
& \leq c_{1}+c_{2}-(q+\lambda)\left(x_{1}+x_{2}+K\right)+\lambda\left(x_{1}+x_{2}+K\right) G\left(\left(x_{1} / b_{1}\right) \wedge\left(x_{2} / b_{2}\right)\right) \\
& \leq c_{1}+c_{2}-q K .
\end{aligned}
$$

Thus there are infinitely many viscosity solutions of the HJB equation (12).

We now show that the optimal value function $V$ can be characterized as the smallest viscosity supersolution of (12) with a suitable growth condition. We say that the function $u: \mathbf{R}_{+}^{2} \rightarrow \mathbf{R}$ satisfies the growth condition, if there exists $K>0$ such that

$$
u(\bar{x}) \leq K+x_{1}+x_{2} \text { for all } \bar{x} \in \mathbf{R}_{+}^{2} .
$$

We have the following result.

Proposition 3.6 Let $\bar{L} \in \Pi_{\bar{x}}$ be any admissible strategy and let $\bar{u}$ be any viscosity supersolution of (12) satisfying growth condition $(\mathrm{G})$, we have that $V_{\bar{L}}(\bar{x}) \leq \bar{u}(x)$.

The proof of this proposition is a straightforward extension to the two-dimensional case of the corresponding proof of Proposition 4.4 in [10], taking again the zero-expectation martingale defined in (13) and using Lemmas 3.1 and 3.2.

From the last proposition and Theorem 3.5, we conclude the following corollary.

Corollary 3.7 The optimal value function $V$ is the smallest viscosity supersolution of (12) satisfying growth condition (G).

Remark 3 From Proposition 3.6 we can deduce the usual viscosity verification result: If the value function $V_{\pi}$ for some strategy $\pi$ or a limit of value functions $\lim _{n \rightarrow \infty} V_{\pi_{n}}$ for some strategies $\pi_{n}$ is a viscosity supersolution of (12), then it is the optimal value function (6). Note that by definition $V$ is a limit of value functions. However, we expect $V$ to be a value function of a family of admissible strategies with some particular structure. This problem will be analyzed in next sections. 


\section{Optimal strategy}

First in this section, we introduce some special families of admissible strategies which depends only on the current surplus levels.

Assuming that $V$ is differentiable in $\mathbf{R}_{+}^{2}$, the Eq. (12) suggests how the dividends are paid depending on the current surplus $\bar{x}=\left(x_{1}, x_{2}\right) \in \mathbf{R}_{+}^{2}$ :

- If the current surplus is in the set

$$
\mathcal{C}^{*}=\left\{\bar{x} \in \mathbf{R}_{+}^{2}: \mathcal{L}(V)(\bar{x})=0,1-V_{x_{1}}(\bar{x})<0,1-V_{x_{2}}(\bar{x})<0\right\}
$$

no dividends are paid.

- If the current surplus is in the set

$$
\mathcal{B}_{0}^{*}=\left\{\bar{x} \in \mathbf{R}_{+}^{2}: \mathcal{L}(V)(\bar{x})<0,1-V_{x_{1}}(\bar{x})=0,1-V_{x_{2}}(\bar{x})=0\right\}
$$

both branches pay a lump sum as dividends.

- If the current surplus is in the set

$$
\mathcal{B}_{1}^{*}=\left\{\bar{x} \in \mathbf{R}_{+}^{2}: \mathcal{L}(V)(\bar{x})<0,1-V_{x_{1}}(\bar{x})=0,1-V_{x_{2}}(\bar{x})<0\right\}
$$

the first branch pays a lump sum as dividends.

- If the current surplus is in the set

$$
\mathcal{B}_{2}^{*}=\left\{\bar{x} \in \mathbf{R}_{+}^{2}: \mathcal{L}(V)(\bar{x})<0,1-V_{x_{1}}(\bar{x})<0,1-V_{x_{2}}(\bar{x})=0\right\}
$$

the second branch pays a lump sum as dividends.

- If the current surplus is in the set

$$
\mathcal{A}_{0}^{*}=\left\{\bar{x} \in \mathbf{R}_{+}^{2}: \mathcal{L}(V)(\bar{x})=0,1-V_{x_{1}}(\bar{x})=0,1-V_{x_{2}}(\bar{x})=0\right\}
$$

both branches pay their incoming premiums as dividends.

- If we define $\mathcal{A}_{1}^{*}$ as the boundary between $\mathcal{B}_{1}^{*}$ and $\mathcal{C}^{*}$, we would have

$$
\mathcal{A}_{1}^{*}=\left\{\bar{x} \in \mathbf{R}_{+}^{2}: \mathcal{L}(V)(\bar{x})=0,1-V_{x_{1}}(\bar{x})=0,1-V_{x_{2}}(\bar{x})<0\right\} .
$$

If the current surplus is in the set $\mathcal{A}_{1}^{*}$, the first branch could pay some part of its incoming premium as dividends.

- Analogously, if we define $\mathcal{A}_{2}^{*}$ as the boundary between $\mathcal{B}_{2}^{*}$ and $\mathcal{C}^{*}$, we would have

$$
\mathcal{A}_{2}^{*}=\left\{\bar{x} \in \mathbf{R}_{+}^{2}: \mathcal{L}(V)(\bar{x})=0,1-V_{x_{1}}(\bar{x})<0,1-V_{x_{2}}(\bar{x})=0\right\} .
$$

If the current surplus is in the set $\mathcal{A}_{2}^{*}$, the second branch could pay some part of its incoming premium as dividends. 
If $V$ is not differentiable at some points, still these sets could be defined in a viscosity sense.

Let us define $\mathcal{M}$ as the half line in $\mathbf{R}_{+}^{2}$ that contains the origin with slope $b_{2} / b_{1}$. Note that, when the current surplus is outside $\mathcal{M}$, there exists the possibility that the ruin occur leaving one branch with positive surplus; if the current surplus is in the set

$$
D^{1}:=\left\{\bar{x} \in \mathbf{R}_{+}^{2}:\left(b_{2} / b_{1}\right) x_{1}>x_{2}\right\},
$$

then the first branch will be the one with eventual positive surplus at ruin time and conversely, if the current surplus is in the set

$$
D^{2}:=\left\{\bar{x} \in \mathbf{R}_{+}^{2}:\left(b_{2} / b_{1}\right) x_{1}<x_{2}\right\},
$$

the same could happen with the second branch.

\subsection{Optimal strategy in $D^{1}$}

Given a initial surplus $\left(x_{1}, x_{2}\right) \in \mathbf{R}_{\text {, }}^{2}$, we define the set $\widehat{\Pi}_{x_{1}, x_{2}} \subset \Pi_{x_{1}, x_{2}}$ in the following way. If $\left(x_{1}, x_{2}\right) \in D^{2} \cup \mathcal{M}$ then $\prod_{x_{1}, x_{2}}$ is the set of all the admissible strategies in which the controlled process remains in the set $D^{2} \cup \mathcal{M}$ until ruin time; if $\left(x_{1}, x_{2}\right) \in$ $D^{1}$, then $\widehat{\Pi}_{x_{1}, x_{2}}$ is the set of all the admissible strategies in which the first branch pays immediately at least $x_{1}-\left(b_{1} / b_{2}\right) x_{2}$ as dividends and afterwards the controlled process remains in the set $D^{2} \cup \mathcal{M}$. From (5), we get

$$
\widehat{\Pi}_{x_{1}, x_{2}}=\left\{\left(L_{1}(t), L_{2}(t)\right) \in \Pi_{x_{1}, x_{2}}: L_{1}(t) \geq x_{1}-\frac{b_{1}}{b_{2}} x_{2}+\left(c_{1}-\frac{b_{1}}{b_{2}} c_{2}\right) t+\frac{b_{1}}{b_{2}} L_{2}(t) \text { for } t \geq 0\right\} .
$$

Proposition 4.1 We have that $V\left(x_{1}, x_{2}\right)=\sup _{\bar{L} \in \hat{\Pi}_{x_{1}, x_{2}}} V_{\bar{L}}\left(x_{1}, x_{2}\right)$.

Proof Given any admissible $\bar{L}^{0}=\left(L_{1}^{0}, L_{2}^{0}\right) \in \Pi_{x_{1}, x_{2}}$, let us define

$$
\bar{L}^{1}(t)=\left(\max \left\{x_{1}-\frac{b_{1}}{b_{2}} x_{2}+\left(c_{1}-\frac{b_{1}}{b_{2}} c_{2}\right) t+\frac{b_{1}}{b_{2}} L_{2}^{0}(t), L_{1}^{0}(t)\right\}, L_{2}^{0}(t)\right) .
$$

We have that $\bar{L}^{1} \in \widehat{\Pi}_{x_{1}, x_{2}}$ because $L_{1}^{1}(t)$ and $L_{2}^{1}(t)$ are predictable, positive and increasing and also

$$
L_{1}^{1}(t) \geq x_{1}-\frac{b_{1}}{b_{2}} x_{2}+\left(c_{1}-\frac{b_{1}}{b_{2}} c_{2}\right) t+\frac{b_{1}}{b_{2}} L_{2}^{1}(t)
$$

Let us prove now that the ruin time $\widehat{\tau}^{L}$ corresponding to the admissible strategy $\bar{L}^{0}$ is less than or equal to $\hat{\tau}^{-1}$. By definition, we have that 


$$
x_{1}+c_{1} t-\sum_{i=1}^{N_{t}} b_{1} U_{i}-L_{1}^{0}(t) \geq 0
$$

and

$$
x_{2}+c_{2} t-\sum_{i=1}^{N_{t}} b_{2} U_{i}-L_{2}^{0}(t) \geq 0
$$

for any $t \leq \widehat{\tau}^{0}$. Thus $X_{2}^{\bar{L}^{1}}(t)=X_{2}^{\bar{L}^{0}}(t) \geq 0$, and

$$
\begin{aligned}
& X_{1}^{\bar{L}^{1}}(t)=x_{1}+c_{1} t-\sum_{i=1}^{N_{t}} b_{1} U_{i}-L_{1}^{1}(t) \\
& =\left\{\begin{array}{c}
X_{1}^{\bar{L}^{0}}(t) \text { if } x_{1}-\frac{b_{1}}{b_{2}} x_{2}+\left(c_{1}-\frac{b_{1}}{b_{2}} c_{2}\right) t+\frac{b_{1}}{b_{2}} L_{2}^{0}(t) \leq L_{1}^{0}(t) \\
\frac{b_{1}}{b_{2}} X_{2}^{\bar{L}^{0}}(t) \text { if } x_{1}-\frac{b_{1}}{b_{2}} x_{2}+\left(c_{1}-\frac{b_{1}}{b_{2}} c_{2}\right) t+\frac{b_{1}}{b_{2}} L_{2}^{0}(t)>L_{1}^{0}(t)
\end{array}\right. \\
& \geq 0
\end{aligned}
$$

for any $t \leq \widehat{\tau}^{0}$.

From (15) we have that $L_{1}^{1}(t)+L_{2}^{1}(t) \geq L_{1}^{0}(t)+L_{2}^{0}(t)$ for $0 \leq t<\widehat{\tau}^{-1}$, so we obtain

$$
V_{\bar{L}^{0}}(\bar{x}) \leq V_{\bar{L}^{1}}(\bar{x})
$$

and the result follows.

Corollary 4.2 If $\left(x_{1}, x_{2}\right) \in D^{1}$, then $V\left(x_{1}, x_{2}\right)=x_{1}-\left(b_{1} / b_{2}\right) x_{2}+V\left(\left(b_{1} / b_{2}\right) x_{2}, x_{2}\right)$.

Proof By (14), we have that $\bar{L}^{0}=\left(L_{1}(t), L_{2}(t)\right) \in \widehat{\Pi}_{x_{1}, x_{2}}$ if and only if

$$
\bar{L}^{1}=\left(L_{1}(t)-x_{1}-\left(b_{1} / b_{2}\right) x_{2}, L_{2}(t)\right) \in \widehat{\Pi}_{\left(b_{1} / b_{2}\right) x_{2}, x_{2}} .
$$

Since we have that $V_{\bar{L}^{0}}\left(x_{1}, x_{2}\right)=x_{1}-\left(b_{1} / b_{2}\right) x_{2}+V_{\bar{L}^{1}}\left(\left(b_{1} / b_{2}\right) x_{2}, x_{2}\right)$, the result follows by Proposition 4.1.

From the last Corollary we can deduce that if $\bar{x} \in D^{1}$ then the optimal value function is a limit of strategies in which the first branch immediately pays $\left(x_{1}-\left(b_{1} / b_{2}\right) x_{2}\right)$ as dividends, so the current surplus goes immediately to $\mathcal{M}$ in the horizontal direction, and afterwards the controlled process remains in $D^{2} \cup \mathcal{M}$ until ruin time. We can ask ourselves if an analogous result holds for $\bar{x} \in D^{2}$, that is whether the optimal 
value function is a limit of strategies in which the second branch immediately pays $x_{2}-\left(b_{2} / b_{1}\right) x_{1}$ as dividends-so the current surplus goes immediately to $\mathcal{M}$ in the vertical direction - and afterwards the controlled process remains in $\mathcal{M}$ until ruin time. We will see in the next section that in general this holds true only in the case in which $c_{2} / c_{1}=b_{2} / b_{1}$.

\section{2 $\mathcal{M}$-strategies}

Given an initial surplus $\left(x_{1}, x_{2}\right) \in \mathbf{R}_{+}^{2}$, we define the set $\widetilde{\Pi}_{x_{1}, x_{2}} \subset \widehat{\Pi}_{x_{1}, x_{2}} \subset \Pi_{x_{1}, x_{2}}$ as the set all the admissible strategies which pay immediately dividends in the following way: the first branch pays immediately $x_{1}-\left(b_{1} / b_{2}\right) x_{2}$ as dividends if $\left(x_{1}, x_{2}\right) \in D^{1} \cup \mathcal{M}$ or the second branch pays immediately $x_{2}-\left(b_{2} / b_{1}\right) x_{1}$ as dividends if $\left(x_{1}, x_{2}\right) \in D^{2}$; afterwards the controlled process remains in the set $\mathcal{M}$ until ruin time.

Let us define

$$
\widetilde{V}\left(x_{1}, x_{2}\right):=\sup _{\bar{L} \in \widetilde{\Pi}_{x_{1}, x_{2}}} V_{\bar{L}}\left(x_{1}, x_{2}\right)
$$

Let us also define $\bar{V}$ as the value function of the best strategy with initial surplus $\left(\left(b_{1} / b_{2}\right) x_{2}, x_{2}\right) \in \mathcal{M}$ whose controlled process remains in the set $\mathcal{M}$ until ruin time, that is

$$
\bar{V}\left(x_{2}\right):=\widetilde{V}\left(\frac{b_{1}}{b_{2}} x_{2}, x_{2}\right)
$$

By definition, we have that

$$
\widetilde{V}\left(x_{1}, x_{2}\right)=\left(x_{1}-\frac{b_{1}}{b_{2}} x_{2}+\bar{V}\left(x_{2}\right)\right) I_{D^{1} \cup \mathcal{M}}\left(x_{1}, x_{2}\right)+\left(x_{2}-\frac{b_{2}}{b_{1}} x_{1}+\bar{V}\left(\frac{b_{2}}{b_{1}} x_{1}\right)\right) I_{D^{2}}\left(x_{1}, x_{2}\right) .
$$

In order to find $\bar{V}$, let us consider the following auxiliary one-dimensional optimization problem. Let $\Pi_{x_{2}}$ be the set of admissible dividend payment strategy corresponding to the one-dimensional compound Poisson process with initial surplus $x_{2} \geq 0$, slope $c_{2}$, claim arrivals given by the Poisson process $N_{t}$ and claim-sizes $b_{2} U_{i}$. Given any $L_{2} \in \Pi_{x_{2}}$, we define

$$
W_{L_{2}}\left(x_{2}\right):=E_{x_{2}}\left(\int_{0}^{\hat{\tau}} e^{-q s}\left(c_{1}-\frac{b_{1}}{b_{2}} c_{2}\right) d s+\left(1+\frac{b_{1}}{b_{2}}\right) \int_{0}^{\hat{\tau}} e^{-q s} d L_{s}^{2}\right),
$$

and

$$
\bar{W}\left(x_{2}\right)=\sup _{L_{2} \in \Pi_{x_{2}}} W_{L_{2}}\left(x_{2}\right) .
$$

Note that, since any $\bar{L}=\left(L_{1}, L_{2}\right) \in \widetilde{\Pi}_{\left(b_{1} / b_{2}\right) x_{2}, x_{2}}$ satisfies 


$$
L_{1}(t)=\frac{b_{1}}{b_{2}} L_{2}(t)+\left(c_{1}-\frac{b_{1}}{b_{2}} c_{2}\right) t
$$

then we have that

$$
V_{\bar{L}}\left(\frac{b_{1}}{b_{2}} x_{2}, x_{2}\right)=W_{L_{2}}\left(x_{2}\right)
$$

Thus $\bar{V}$ and $\bar{W}$ coincide which is stated formally in next proposition.

Proposition 4.3 For any $x_{2} \geq 0$ we have $\bar{V}\left(x_{2}\right)=\bar{W}\left(x_{2}\right)$.

We study now the optimization problem (18). The function $\bar{W}$ can be seen as the optimal value function (up to a constant) of the dividend problem with a reward for avoiding early ruin; see for instance Thonhauser and Albrecher [38]. In effect, we can write

$$
\frac{\bar{W}\left(x_{2}\right)}{1+\frac{b_{1}}{b_{2}}}=\sup _{L_{2} \in \Pi_{x_{2}}} E_{x_{2}}\left(\int_{0}^{\tau} e^{-q s} \kappa d s+\int_{0}^{\tau} e^{-q s} d L_{s}^{2}\right),
$$

where

$$
\kappa=\frac{c_{1}-\left(b_{1} / b_{2}\right) c_{2}}{1+b_{1} / b_{2}}
$$

From [38], we have that $\bar{W}$ has the following associated HJB equation,

$$
\max \left\{1+\frac{b_{1}}{b_{2}}-\bar{W}^{\prime}\left(x_{2}\right), \overline{\mathcal{L}}(\bar{W})\left(x_{2}\right)\right\}=0,
$$

where

$$
\overline{\mathcal{L}}(\bar{W})\left(x_{2}\right):=c_{2} \bar{W}^{\prime}\left(x_{2}\right)-(\lambda+q) \bar{W}\left(x_{2}\right)+\lambda \int_{0}^{x_{2} / b_{2}} \bar{W}\left(x_{2}-b_{2} \alpha\right) d G(\alpha)+c_{1}-\frac{b_{1}}{b_{2}} c_{2} .
$$

We can state the following proposition for the optimal one-dimensional reward problem. The proof is similar to the one-dimensional optimization dividend problem without reward (see for instance Azcue and Muler [10], Propositions 3.1 and 4.4). 
Proposition 4.4 $\bar{W}$ is a locally Lipschitz viscosity solution of (19). Moreover, it is the smallest viscosity solution of this equation for all $x_{2}>0$ satisfying the growth condition $\bar{W}\left(x_{2}\right) \leq K+\left(1+b_{1} / b_{2}\right) x_{2}$ for some $K>0$.

From the previous proposition, we can deduce the following verification result: Given a family of admissible strategies $\bar{\pi}=\left\{L_{2}^{x_{2}} \in \Pi_{x_{2}}\right.$ for any $\left.x_{2} \geq 0\right\}$, we define the value function $W_{\bar{\pi}}: \mathbf{R}_{+} \rightarrow \mathbf{R}_{+}$as $W_{\bar{\pi}}\left(x_{2}\right)=W_{L_{2}^{x_{2}}}\left(x_{2}\right)$. If the function $W_{\bar{\pi}}\left(x_{2}\right)$ is a vis-

cosity supersolution of (19) for each $x_{2}>0$, then $W_{\bar{\pi}}$ coincides with $\bar{W}$.

We also have the following result about the optimal dividend strategy of the problem (18), the proof is similar to the case without reward, see for instance Azcue and Muler [10], Sections 5.1 and 5.2.

Proposition 4.5 The dividend strategy that optimizes (18) is a band dividend strategy depending on the sets $\overline{\mathcal{A}}, \overline{\mathcal{B}}$ and $\overline{\mathcal{C}}$, where

$$
\overline{\mathcal{A}}:=\left\{x_{2} \geq 0:\left(c_{1}+c_{2}\right)-(\lambda+q) \bar{W}\left(x_{2}\right)+\lambda \int_{0}^{x_{2} / b_{2}} \bar{W}\left(x_{2}-b_{2} \alpha\right) d G(\alpha)=0\right\}
$$

is closed and bounded,

$$
\overline{\mathcal{B}}:=\left\{x_{2} \geq 0: \bar{W}^{\prime}\left(x_{2}\right)=1+\frac{b_{1}}{b_{2}},\left(c_{1}+c_{2}\right)-(\lambda+q) \bar{W}\left(x_{2}\right)+\lambda \int_{0}^{x_{2} / b_{2}} \bar{W}\left(x_{2}-b_{2} \alpha\right) d G(\alpha)<0\right\}
$$

is left-open,

$$
\overline{\mathcal{C}}=\mathbf{R}_{+}-\overline{\mathcal{A}} \cup \overline{\mathcal{B}}
$$

is right-open, and there exists $\widetilde{x}_{2}$ such that $\left(\widetilde{x}_{2}, \infty\right) \subset \overline{\mathcal{B}}$. We have the following cases:

- if the current surplus is in $\overline{\mathcal{A}}$ the incoming premium $c_{2}$ is paid as dividends;

- if the current surplus is in $\overline{\mathcal{B}}$ a positive amount of money is paid as dividends in order to bring the surplus process back to $\overline{\mathcal{A}}$;

- if the surplus is in $\overline{\mathcal{C}}$, no dividends are paid.

Let us go back to problem (16) in $\mathcal{M}$. Using that

$$
\widetilde{V}\left(\frac{b_{1}}{b_{2}} x_{2}, x_{2}\right)=\bar{V}\left(x_{2}\right)=\bar{W}\left(x_{2}\right),
$$


we can split the half line $\mathcal{M}$ into three sets:

$$
\begin{aligned}
& \mathcal{A}_{\mathcal{M}}:=\left\{\left(\frac{b_{1}}{b_{2}} x_{2}, x_{2}\right): x_{2} \in \overline{\mathcal{A}}\right\}, \\
& \mathcal{B}_{\mathcal{M}}:=\left\{\left(\frac{b_{1}}{b_{2}} x_{2}, x_{2}\right): x_{2} \in \overline{\mathcal{B}}\right\},
\end{aligned}
$$

and

$$
\mathcal{C}_{\mathcal{M}}:=\left\{\left(\frac{b_{1}}{b_{2}} x_{2}, x_{2}\right): x_{2} \in \overline{\mathcal{C}}\right\}
$$

From Proposition 4.5 we conclude that the optimal dividend strategy for the auxiliary problem (16) with initial surplus in $\mathcal{M}$ is the following:

- if $\left(\left(b_{1} / b_{2}\right) x_{2}, x_{2}\right) \in \mathcal{A}_{\mathcal{M}}$ both branches pay the incoming premium as dividends until next claim,

- if $\left(\left(b_{1} / b_{2}\right) x_{2}, x_{2}\right) \in \mathcal{B}_{\mathcal{M}}$, the second branch pays a positive amount $m$ of money and the first branch pays $\left(b_{1} / b_{2}\right) m$, where $m$ is the minimal amount that brings the surplus process back to $\mathcal{A}_{\mathcal{M}}$,

- and finally if $\left(\left(b_{1} / b_{2}\right) x_{2}, x_{2}\right) \in \mathcal{C}_{\mathcal{M}}$, the second branch pays no dividends and the first branch pays $\left(c_{1}-\left(b_{1} / b_{2}\right) c_{2}\right)$ as dividend rates.

Remark 4 If the optimal value function $V$ coincides with the function $\widetilde{V}$ defined in (16), the optimal strategy would be given by

$$
\begin{aligned}
& \mathcal{B}_{1}^{*}=\left\{\bar{x} \in D^{1}: x_{2} \notin \mathcal{B}_{\mathcal{M}}\right\}, \mathcal{B}_{2}^{*}=\left\{\bar{x} \in D^{2}:\left(b_{2} / b_{1}\right) x_{1} \notin \mathcal{B}_{\mathcal{M}}\right\}, \\
& \mathcal{B}_{0}^{*}=\left(D^{1}-\mathcal{B}_{1}^{*}\right) \cup \mathcal{B}_{\mathcal{M}} \cup\left(D^{2}-\mathcal{B}_{2}^{*}\right),
\end{aligned}
$$

$\mathcal{A}_{0}^{*}=\mathcal{A}_{\mathcal{M}}, \mathcal{A}_{1}^{*}=\mathcal{C}_{\mathcal{M}}$ and $\mathcal{A}_{2}^{*}=\mathcal{C}^{*}=\varnothing$. Moreover,

- in $\mathcal{A}_{0}^{*}$ both the incoming premiums are paid as dividends;

- in $\mathcal{A}_{1}^{*}$ the first branch pays dividends at rate $c_{1}-\left(b_{1} / b_{2}\right) c_{2}$ (and so the surplus process remains in $\mathcal{M}$ );

- in $D^{1}$ the first branch pays immediately $\left(x_{1}-\left(b_{1} / b_{2}\right) x_{2}\right)$, so the current surplus goes immediately to $\mathcal{M}$ in the horizontal direction;

- in $D^{2}$ the second branch pays immediately $\left(x_{2}-\left(b_{2} / b_{1}\right) x_{1}\right)$, so the current surplus goes immediately to $\mathcal{M}$ in the vertical direction.

In the particular case that $c_{1}=\left(b_{1} / b_{2}\right) c_{2}$, the first branch does not need to pay dividends in $\mathcal{C}_{\mathcal{M}}$ to remain in $\mathcal{M}$, so $\mathcal{C}^{*}=\mathcal{A}_{1}^{*}=\mathcal{C}_{\mathcal{M}}$. 


\section{3 $\widetilde{V}$ is the optimal value function in the case $c_{2} / c_{1}=b_{2} / b_{1}$}

In the next propositions we prove that the optimal value function $V$ coincides with the function $\widetilde{V}$ defined in (16).

Proposition 4.6 For any $x_{2} \geq 0$ we have that $V\left(\left(b_{1} / b_{2}\right) x_{2}, x_{2}\right)=\bar{V}\left(x_{2}\right)=\bar{W}\left(x_{2}\right)$.

Proof Given any admissible $\bar{L}^{0}=\left(L_{1}^{0}, L_{2}^{0}\right) \in \Pi_{\left(b_{1} / b_{2}\right) x_{2}, x_{2}}$, let us define $\bar{L}^{1} \in \Pi_{\left(b_{1} / b_{2}\right) x_{2}, x_{2}}$ as

$$
\bar{L}^{1}(t)=\left(L_{1}^{0}(t)+L_{2}^{0}(t)\right)\left(b_{1}, b_{2}\right)
$$

Note that $L_{1}^{1}(t)+L_{2}^{1}(t)=L_{1}^{0}(t)+L_{2}^{0}(t)$ and $\bar{X}^{L^{1}}(t) \in \mathcal{M}$ for $t<\hat{\tau}^{\bar{L}^{1}}$, so $\bar{L}^{1} \in \widetilde{\Pi}_{\left(b_{1} / b_{2}\right) x_{2}, x_{2}}$. It is easy to check that the ruin time $\widehat{\tau}^{L^{0}}$ corresponding to the admissible strategy $\bar{L}^{0}$ is less than or equal to $\widehat{\tau}^{-1}$. Therefore,

$$
V_{\vec{L}^{0}}\left(\frac{b_{1}}{b_{2}} x_{2}, x_{2}\right) \leq V_{\bar{L}^{1}}\left(\frac{b_{1}}{b_{2}} x_{2}, x_{2}\right)
$$

and thus the result follows.

Proposition 4.7 The optimal value function $V=\widetilde{V}$.

Proof By Proposition 4.6 and (4.3) we have that $V=\widetilde{V}$ in $\mathcal{M}$. Then, by Proposition 4.2 we obtain that $V=\widetilde{V}$ in $D^{1}$. The result in $D^{2}$ follows by symmetry.

As a consequence of the previous proposition, the optimal strategy is the one described in Remark 4. As an example, consider the claim-size distribution gamma

$$
G(x)=1-\left(1+\frac{x}{2}\right) e^{-\frac{x}{2}},
$$

the parameters $b_{1}=b_{2}=0.5, \quad c_{1}=c_{2}=21.4, q=0.1$ and $\lambda=10$. In Section 6.2 .1 of [10], it is shown that $\overline{\mathcal{A}}=\{0,10.22\}, \overline{\mathcal{B}}=(0,1.803] \cup(10.22, \infty)$ and $\bar{C}=(1.803,10.22)$, the optimal strategy is depicted in Fig. 1.

Remark $5 \mathrm{~V}$ is not differentiable in $C_{\mathcal{M}}$ because if it were differentiable at a point $\left(\left(b_{1} / b_{2}\right) x_{2}, x_{2}\right) \in C_{\mathcal{M}}$, then both $V_{x_{1}}\left(\left(b_{1} / b_{2}\right) x_{2}, x_{2}\right)$ and $V_{x_{2}}\left(\left(b_{1} / b_{2}\right) x_{2}, x_{2}\right)$ should be one; then

$$
\bar{W}\left(x_{2}\right)=1+\frac{b_{1}}{b_{2}}
$$




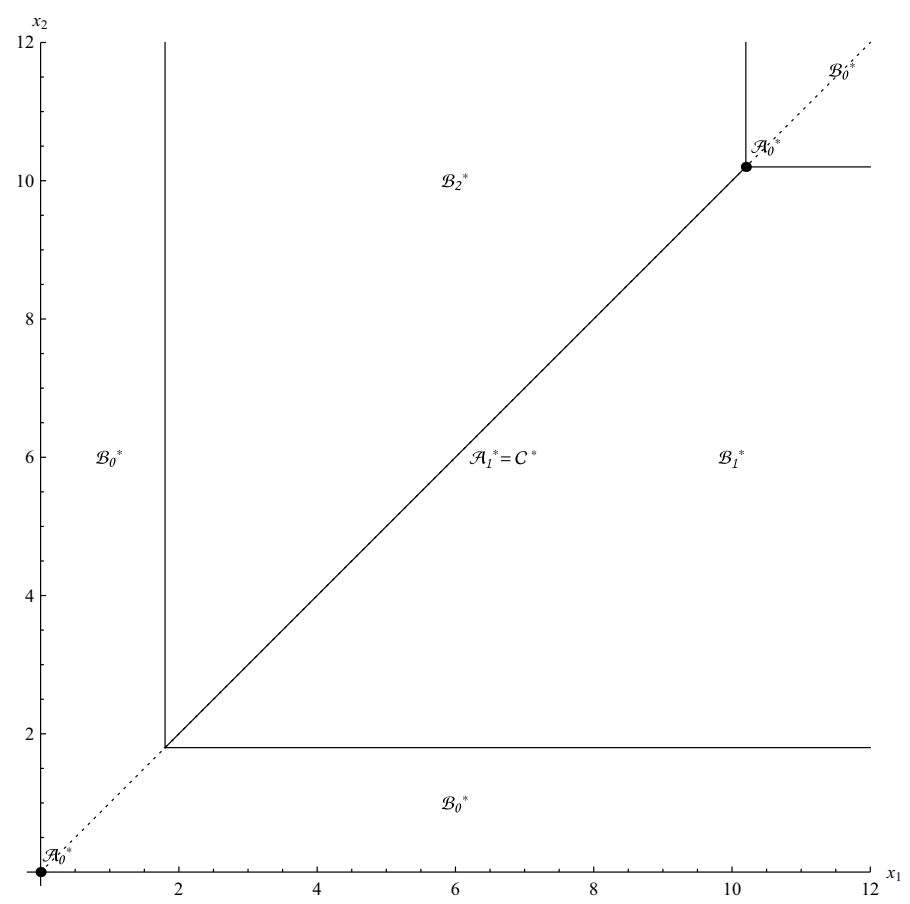

Fig. 1 Optimal strategy for the claim-size distribution gamma

that implies $x_{2} \notin \bar{C}$ which is a contradiction.

\section{4 $\widetilde{V}$ is not the optimal value function in the case $c_{2} / c_{1}<b_{2} / b_{1}$}

In the next proposition we show, that except in a very special case, the function $\widetilde{V}$ defined in (16) is never the optimal value function of the optimization problem (6).

Proposition 4.8 In the case that $\mathcal{C}_{\mathcal{M}}$ defined in (20) is not empty, the function $\widetilde{V}$ is not a viscosity solution of (12) at all the points in the first quadrant.

Proof Let us take any point $\left(x_{1}^{0}, x_{2}^{0}\right) \in \mathcal{C}_{\mathcal{M}}$ with $\underline{x}_{2}^{0}=\left(b_{2} / b_{1}\right) x_{1}^{0}$ such that $\bar{W}$ is differentiable at $x_{2}^{0}$ (since $\bar{W}$ is locally Lipschitz and $\overline{\mathcal{C}}$ is right-open, the set of points in $\overline{\mathcal{C}}$ where $\bar{W}^{\prime}$ exists has full measure). By definition of $\bar{C}$,

$$
\bar{W}^{\prime}\left(x_{2}^{0}\right)-1-\frac{b_{1}}{b_{2}}>0,
$$


and

$$
\overline{\mathcal{L}}(\bar{W})\left(x_{2}^{0}\right)=c_{2} \bar{W}^{\prime}\left(x_{2}^{0}\right)-(\lambda+q) \bar{W}\left(x_{2}^{0}\right)+\lambda \int_{0}^{x_{2}^{0} / b_{2}} \bar{W}\left(x_{2}^{0}-b_{2} \alpha\right) d G(\alpha)+c_{1}-\frac{b_{1}}{b_{2}} c_{2}=0 .
$$

This implies that for any $x_{2}>x_{2}^{0}\left(\right.$ and so $\left.\left(x_{1}^{0}, x_{2}\right) \in D^{2}\right)$ we have,

$$
\widetilde{V}\left(x_{1}^{0}, x_{2}\right)=x_{2}-x_{2}^{0}+\bar{W}\left(x_{2}^{0}\right)=x_{2}-\frac{b_{2}}{b_{1}} x_{1}^{0}+\bar{W}\left(\frac{b_{2}}{b_{1}} x_{1}^{0}\right)
$$

and so it is differentiable at all these points with

$$
\widetilde{V}_{x_{1}}\left(x_{1}^{0}, x_{2}\right)=-\frac{b_{2}}{b_{1}}+\frac{b_{2}}{b_{1}} \bar{W}^{\prime}\left(x_{2}^{0}\right)>1 \text { and } \widetilde{V}_{x_{2}}\left(x_{1}^{0}, x_{2}\right)=1 \text {. }
$$

Then, since $b_{1} c_{2}<b_{2} c_{1}$ and $x_{2} / b_{2}>x_{2}^{0} / b_{2}=x_{1}^{0} / b_{1}$,

$$
\begin{aligned}
\mathcal{L}(\widetilde{V})\left(x_{1}^{0}, x_{2}\right)= & c_{1}\left(-\frac{b_{2}}{b_{1}}+\frac{b_{2}}{b_{1}} \bar{W}^{\prime}\left(x_{2}^{0}\right)\right)+c_{2}-(q+\lambda)\left(x_{2}-x_{2}^{0}+\bar{W}\left(x_{2}^{0}\right)\right) \\
& +\lambda \int_{0}^{x_{2}^{0} / b_{2}}\left(x_{2}-x_{2}^{0}+\bar{W}\left(x_{2}^{0}-b_{2} \alpha\right)\right) d G(\alpha) \\
= & \overline{\mathcal{L}}(\bar{W})\left(x_{2}^{0}\right)+\left(\frac{c_{1} b_{2}}{b_{1}}-c_{2}\right)\left(\bar{W}^{\prime}\left(x_{2}^{0}\right)-1-\frac{b_{1}}{b_{2}}\right)-\left(x_{2}-x_{2}^{0}\right)(q+\lambda) \\
& +\lambda\left(x_{2}-x_{2}^{0}\right) G\left(x_{2}^{0} / b_{2}\right) .
\end{aligned}
$$

Hence, taking $x_{2}$ close enough to $x_{2}^{0}>0$ we get $\mathcal{L}(\widetilde{V})\left(x_{1}^{0}, x_{2}\right)>0$. So, $\widetilde{V}$ is not a viscosity solution of (12) at $\left(x_{1}^{0}, x_{2}\right)$.

Remark 6 In the case that $C_{\mathcal{M}}$ is empty, $A_{\mathcal{M}}=\{(0,0)\}$ and $B_{\mathcal{M}}=M-\{(0,0)\}$, so $W\left(x_{2}\right)=\left(\frac{b_{1}}{b_{2}}+1\right) x_{2}+\frac{c_{1}+c_{2}}{\lambda+q}$ and $\widetilde{V}\left(x_{1}, x_{2}\right)=x_{1}+x_{2}+\frac{c_{1}+c_{2}}{\lambda+q}$.

This strategy is called "take the money and run". Depending on the parameters, this strategy could be or not the optimal one.

The optimal value function $V$ and the optimal strategy for surpluses in $D^{2} \cup \mathcal{M}$, unlike the case $c_{1} / b_{1}=c_{2} / b_{2}$, cannot be obtained in terms of the one-dimensional auxiliary optimization problem as before. We do not have any theoretical result on the 
existence and structure of the optimal strategy in $D^{2} \cup \mathcal{M}$. In Section 5 we will use a two-dimensional numerical scheme to approximate the optimal strategy instead.

\section{Numerical scheme in $D^{2} \cup \mathcal{M}$}

In this section we present a convergent numerical scheme to approximate the optimal value function for the case $c_{1} / b_{1}>c_{2} / b_{2}$ in $\mathbf{R}_{+}^{2}$. In fact, by Corollary 4.2 , we have that the value function satisfy

$$
V\left(x_{1}, x_{2}\right)=x_{1}-\left(b_{1} / b_{2}\right) x_{2}+V\left(\left(b_{1} / b_{2}\right) x_{2}, x_{2}\right)
$$

and so it is enough to approximate the optimal value function in $D^{2} \cup \mathcal{M}$. This numerical scheme can be seen as a particular case of the one described in [11] for the case that the joint multivariate distribution function of the downward jumps is given by $F(x, y):=G\left(\left(x / b_{1}\right) \wedge\left(y / b_{2}\right)\right)$ and both the penalty and the switch-value functions are identically zero.

Given any $\delta>0$, consider $\Delta x_{1}=c_{1} \delta$ and $\Delta x_{2}=c_{2} \delta$, define the grid domain $\mathcal{G}^{\delta}$ in $\mathbf{R}_{+}^{2}$ as

$$
\mathcal{G}^{\delta}:=\left\{\left(n \Delta x_{1}, m \Delta x_{2}\right): n, m \geq 0\right\} .
$$

We look, at each point of the grid $\mathcal{G}^{\delta}$, for the best local strategy among the ones suggested by the operators of the HJB equation (12); these possible local strategies are: the first branch pays a lump sum as dividends, the second one pays a lump sum as dividends, or none of the branches pay dividends. These local strategies are modified in such a way that the controlled surplus after applying these local strategies lies in the grid. The possible control actions at any point of the grid $\mathcal{G}^{\delta}$ are defined as follows: let $\left(n \Delta x_{1}, m \Delta x_{2}\right) \in \mathcal{G}^{\delta}$ be the initial surplus and $\tau$ and $U$ be the time and size of the first claim respectively.

1. $\mathbf{E}_{1}$ : The first branch pays immediately $\Delta x_{1}=c_{1} \delta$ as dividends, so the controlled surplus becomes $\left((n-1) \Delta x_{1}, m \Delta x_{2}\right) \in \mathcal{G}^{\delta}$. The control action $\mathbf{E}_{1}$ can only be applied for $n>0$.

2. $\mathbf{E}_{2}$ : The second branch pays immediately $\Delta x_{2}=c_{2} \delta$ as dividends, so the controlled surplus becomes $\left(n \Delta x_{1},(m-1) \Delta x_{2}\right) \in \mathcal{G}^{\delta}$. The control action $\mathbf{E}_{2}$ can only be applied for $m>0$.

3. $\mathbf{E}_{0}$ : Pay no dividends up to the time $\delta \wedge \tau$. In the case that $\delta<\tau$, the uncontrolled surplus at time $\delta$ is $\left((n+1) \Delta x_{1},(m+1) \Delta x_{2}\right) \in \mathcal{G}^{\delta}$; and if $\delta \geq \tau$, the uncontrolled surplus at time $\tau$ is $\left(n \Delta x_{1}+c_{1} \tau-b_{1} U, m \Delta x_{2}+c_{2} \tau-b_{2} U\right)$. If this vector is in the first quadrant, the branches pay immediately the minimum amount of dividends in such a way that the controlled surplus lies in a point of the grid; this end surplus can be written as

$$
\left(\left[\frac{n \Delta x_{1}+c_{1} \tau-b_{1} U}{\Delta x_{1}}\right] \Delta x_{1},\left[\frac{m \Delta x_{2}+c_{2} \tau-b_{2} U}{\Delta x_{2}}\right] \Delta x_{2}\right) \in \mathcal{G}^{\delta}
$$


the amount paid as dividends for the first and second branch at time $\tau$ is equal to

$$
\begin{aligned}
\Delta \bar{L}(\tau)= & \left(n \Delta x_{1}+c_{1} \tau-b_{1} U-\left[\frac{n \Delta x_{1}+c_{1} \tau-b_{1} U}{\Delta x_{1}}\right] \Delta x_{1}, m \Delta x_{2}+c_{2} \tau-b_{2} U\right) \\
& \left.-\left[\frac{m \Delta x_{2}+c_{2} \tau-b_{2} U}{\Delta x_{2}}\right] \Delta x_{2}\right) .
\end{aligned}
$$

In the case that the end surplus $\left(n \Delta x_{1}+c_{1} \tau-b_{1} U, m \Delta x_{2}+c_{2} \tau-b_{2} U\right)$ is not in the first quadrant, $\tau$ is the ruin time.

For technical reasons, an extra control action $\mathbf{E}_{s}$ is considered, under this control action no more dividends are paid. The set of control actions is denoted by $\mathcal{E}=\left\{\mathbf{E}_{1}, \mathbf{E}_{2}, \mathbf{E}_{0}, \mathbf{E}_{s}\right\}$.

Define $\prod_{n \Delta x_{1}, m \Delta x_{2}}^{\delta} \subset \Pi_{n \Delta x_{1}, m \Delta x_{2}}$ as the set of all the admissible dividend strategies

with initial surplus $\left(n \Delta x_{1}, m \Delta x_{2}\right) \in \mathcal{G}^{\delta}$ which can be obtained by a sequence (finite or infinite) of control actions in $\mathcal{E}$ at each point of the grid. The $\delta$-optimal function $V^{\delta}$ is defined at each point $\left(n \Delta x_{1}, m \Delta x_{2}\right) \in \mathcal{G}^{\delta}$ as the supremum of the value functions of admissible strategies which are combination of the control actions in $\mathcal{E}$, that is

$$
V^{\delta}\left(n \Delta x_{1}, m \Delta x_{2}\right)=\sup _{\pi \in \Pi_{n \Delta x_{1}, m \Delta x_{2}}^{\delta}} V_{\pi}\left(n \Delta x_{1}, m \Delta x_{2}\right) .
$$

Note that the extra control action $\mathbf{E}_{s}$ corresponds in the numerical scheme described in [11] to switch immediately to a switch-value function identically 0 (which is always smaller than $V$ ), so it is never optimal to use this control; however -as we will see later on- it is used to find a simple admissible dividends strategy in $\Pi_{n \Delta x_{1}, m \Delta x_{2}}^{\delta}$ to start a recursive construction in $\Pi_{n \Delta x_{1}, m \Delta x_{2}}^{\delta}$ which will converge to $V^{\delta}$.

Define $v^{\delta}(n, m):=V^{\delta}\left(n \Delta x_{1}, m \Delta x_{2}\right)$. It is proved in [11], that the function $v^{\delta}$ is a solution of the following discrete version of the HJB equation

$$
\max \left\{T_{0}(W)-W, T_{1}(W)-W, T_{2}(W)-W\right\}=0
$$

at $(n, m) \in \mathbf{N}_{0}^{2}$. Here the operators $T_{0}, T_{1}$ and $T_{2}$ are defined as

$$
\begin{aligned}
& T_{1}(W)(n, m):=W(n-1, m)+\Delta x_{1}, \\
& T_{2}(W)(n, m):=W(n, m-1)+\Delta x_{2},
\end{aligned}
$$

and

$$
T_{0}(W)(n, m):=W(n+1, m+1) e^{-(q+\lambda) \delta}+\mathcal{I}^{\delta}(W)(n, m)
$$

where 


$$
\begin{aligned}
& \mathcal{I}^{\delta}(W)(n, m) \\
& =\int_{0}^{\delta}\left(\int_{\overline{0}}^{\frac{n \Delta x_{1}+c_{1} t}{b_{1}} \wedge \frac{m \Delta x_{2}+c_{2} t}{b_{2}}} e^{-q t} W\left(\left[\frac{n \Delta x_{1}+c_{1} t-b_{1} \alpha}{\Delta x_{1}}\right],\left[\frac{m \Delta x_{2}+c_{2} t-b_{2} \alpha}{\Delta x_{2}}\right]\right) d G(\alpha)\right) \lambda e^{-\lambda t} d t
\end{aligned}
$$

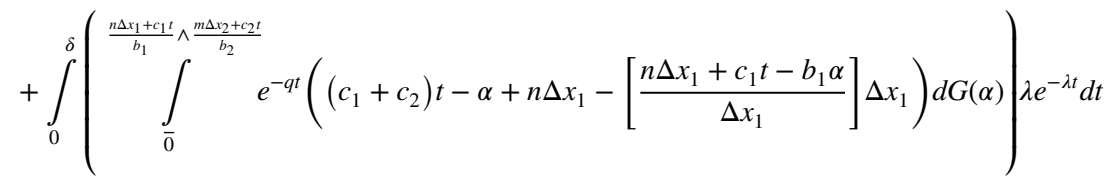

$$
\begin{aligned}
& \left.+\int_{0}^{\delta} \int_{\overline{0}}^{\frac{n \Delta x_{1}+c_{1} t}{b_{1}} \wedge \frac{m \Delta x_{2}+c_{2} t}{b_{2}}} e^{-q t}\left(m \Delta x_{2}-\left[\frac{m \Delta x_{2}+c_{2} t-b_{2} \alpha}{\Delta x_{2}}\right] \Delta x_{2}\right) d G(\alpha)\right) \lambda e^{-\lambda t} d t .
\end{aligned}
$$

Remark 7 Analogously to the Remark 2 for the HJB equation (12), there are infinitely many solutions of the discrete HJB equation; in fact all the functions $u(n, m)=n \Delta x_{1}+m \Delta x_{2}+K$ are solutions of (22) for $K$ large enough. Indeed,

$$
\left(T_{0}(W)-W\right)(n, m) \leq\left(c_{1}+c_{2}\right)\left(\delta e^{-(q+\lambda) \delta}+\frac{\lambda}{(\lambda+q)^{2}}\left(1-e^{-(\lambda+q) \delta}\right)-K\left(1-e^{-(\lambda+q) \delta}\right) \frac{q}{\lambda+q} .\right.
$$

Consider the operator $T$ defined as

$$
T:=\max \left\{T_{0}, T_{1}, T_{2}\right\} .
$$

Using the results in [11], we have that:

- $\quad v^{\delta}$ can be characterized as the smallest solution of the discrete HJB equation (22). Also, if a family of strategies $\tilde{\pi}=\left(\pi_{(n, m)}\right)_{(n, m) \in \mathbf{N}_{0}^{2}}$ with $\pi_{(n, m)} \in \Pi_{n \Delta x_{1}, m \Delta x_{2}}^{\delta}$ satisfy that the function $u(n, m):=V_{\pi_{(n, m)}}\left(n \Delta x_{1}, m \Delta x_{2}\right)$ is a solution of the discrete HJB equation (22) for all $(n, m) \in \mathbf{N}_{0}^{2}$ then $u=v^{\delta}$ and so $V^{\delta}\left(n \Delta x_{1}, m \Delta x_{2}\right)=V_{\pi_{(n, m)}}\left(n \Delta x_{1}, m \Delta x_{2}\right)$ for $(n, m) \in \mathbf{N}_{0}^{2}$.

- There exists an optimal admissible strategy for the problem (21) at any point of the grid. This strategy, called the $\delta$-optimal strategy, is stationary in the sense that the control actions depend only on which point of the grid the current surplus lies. In the case that $T_{1}\left(v^{\delta}\right)(n, m)-v^{\delta}(n, m)=0$, the optimal control action at the point $\left(n \Delta x_{1}, m \Delta x_{2}\right) \in \mathcal{G}^{\delta}$ is $\mathbf{E}_{1}$; in the case that 
$T_{2}\left(v^{\delta}\right)(n, m)-v^{\delta}(n, m)=0$, the optimal control action is $\mathbf{E}_{2}$; and finally in the case that $T_{0}\left(v^{\delta}\right)(n, m)-v^{\delta}(n, m)=0$, the optimal control action is $\mathbf{E}_{0}$.

- The function $v^{\delta}$ can be obtained recursively. The operator $T$ defined in (26) is increasing and $v^{\delta}$ is a fixed point of this operator. However, by Remark 7, this operator has infinitely many fixed points. Since $T$ is not a contraction operator, $v^{\delta}$ can not be obtained numerically as a fixed point; so we construct value functions $v_{l}^{\delta}(n, m):=V_{\pi_{(n, m)}^{l}}\left(n \Delta x_{1}, m \Delta x_{2}\right)$ of strategies $\pi_{(n, m)}^{l} \in \Pi_{n \Delta x_{1}, m \Delta x_{2}}^{\delta}$ which can be calculated explicitly such that $v_{l}^{\delta} \nearrow v^{\delta}$ as $l \rightarrow \infty$. In order to do that, let us define iteratively families of strategies $\tilde{\pi}_{l}=\left(\pi_{(n, m)}^{l}\right)_{(n, m) \in \mathrm{N}_{0}^{2}}$ for each $l \geq 1$ in the following way;

1. Start with the family of strategies $\tilde{\pi}_{1}=\left(\pi_{(n, m)}^{1}\right)_{(n, m) \in \mathbf{N}_{0}^{2}}$ where $\pi_{(n, m)}^{1} \in \Pi_{n \Delta x_{1}, m \Delta x_{2}}^{\delta}$ corresponds to the local control $\mathbf{E}_{s}$ (not more dividends are paid); the value function of this strategy is $v_{1}^{\delta}(n, m):=V_{\pi_{(n, m)}^{1}}\left(n \Delta x_{1}, m \Delta x_{2}\right)=0$

2. Given $\tilde{\pi}_{l}=\left(\pi_{(n, m)}^{l}\right)_{(n, m) \in \mathbf{N}_{0}^{2}}$ with $\pi_{(n, m)}^{l} \in \Pi_{n \Delta x_{1}, m \Delta x_{2}}^{\delta}, \quad$ define $\tilde{\pi}_{l+1}=\left(\pi_{(n, m)}^{l+1}\right)_{(n, m) \in \mathbf{N}_{0}^{2}}$ as follows, for any $(n, m) \in \mathbf{N}_{0}^{2}$, the best strategy $\pi_{(n, m)}^{l+1} \in \Pi_{n \Delta x_{1}, m \Delta x_{2}}^{\delta}$ is chosen among the ones which initially follows one of

the control actions in the set $\mathcal{E}$ and then continues with the strategy in the family $\tilde{\pi}_{l}$ at the end point of the best control action. The value function of $\pi_{(n, m)}^{l+1}$ is given by

$$
v_{l+1}^{\delta}(n, m):=V_{\pi_{(n, m)}^{l+1}}\left(n \Delta x_{1}, m \Delta x_{2}\right)=T\left(v_{l}^{\delta}\right)(n, m)=T^{(l)}\left(v_{1}^{\delta}\right)(n, m) \text { for }(n, m) \in \mathbf{N}_{0}^{2} .
$$

Finally, since $T$ is increasing, we have that $v_{l+1}^{\delta} \geq v_{l}^{\delta}$ for any $l \geq 1$ and so there exists $\bar{v}=\lim _{l \rightarrow \infty} v_{l}^{\delta}$. The function $\bar{v}$ is a solution of the discrete HJB equation (22) and it is constructed as a value function of a combination of local controls in $\mathcal{E}$, then $\bar{v}=v^{\delta}$.

- Let us extend the definition of $V^{\delta}$ from $\mathcal{G}^{\delta}$ to all the points in the first quadrant as

$$
V^{\delta}\left(x_{1}, x_{2}\right)=V^{\delta}\left(\left[\frac{x_{1}}{\Delta x_{1}}\right] \Delta x_{1},\left[\frac{x_{2}}{\Delta x_{2}}\right] \Delta x_{2}\right)+x_{1}-\left[\frac{x_{1}}{\Delta x_{1}}\right] \Delta x_{1}+x_{2}-\left[\frac{x_{2}}{\Delta x_{2}}\right] \Delta x_{2} .
$$

Then, $V^{\delta}\left(x_{1}, x_{2}\right)$ is the value function of the admissible strategy in $\Pi_{x_{1}, x_{2}}$ in which the first and second branch pay immediately $x_{1}-\left[x_{1} / \Delta x_{1}\right] \Delta x_{1}$ and 


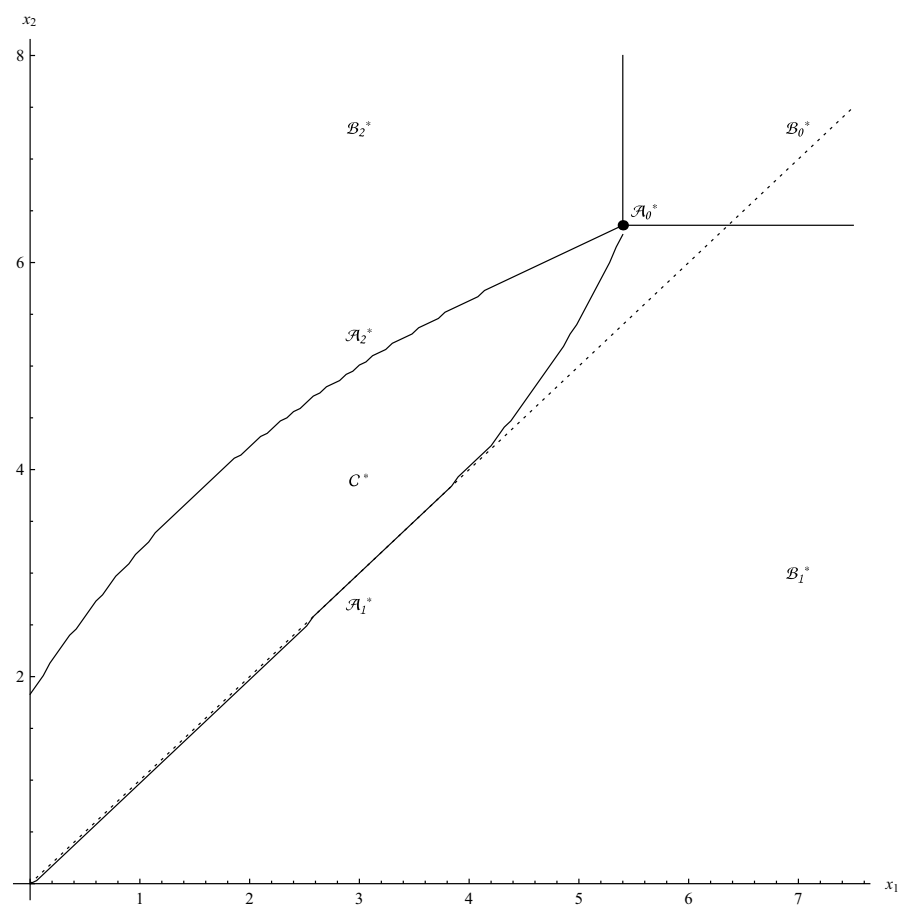

Fig. $2 \delta$-optimal strategy with $\delta=0.03$

$x_{2}-\left[x_{2} / \Delta x_{2}\right] \Delta x_{2} \quad$ respectively as dividends and then follow $\pi_{(n, m)} \in \Pi_{\left[x_{1} / \Delta x_{1}\right] \Delta x_{1},\left[x_{2} / \Delta x_{2}\right] \Delta x_{2}}^{\delta}$. For any $\delta>0$, it holds that $V^{\delta / 2^{k}} \nearrow V$ locally uniformly in the first quadrant as $k$ goes to infinity. The grids $\mathcal{G}^{\delta / 2^{k}}$ are taken in order to have $\mathcal{G}^{\delta / 2^{k}} \subset \mathcal{C}^{\delta / 2^{k+1}}$ and so $V^{\delta / 2^{k}} \leq V^{\delta / 2^{k+1}}$.

\section{Numerical examples}

We show three numerical examples with parameters $b_{1}=b_{2}=0.5, c_{1}=2$, $c_{2}=1, q=0.05$ and $\lambda=1$ and different claim-size distributions $G$. We use the numerical scheme introduced in Section 5 and obtain the numerical approximation $V^{\delta}$ of the optimal value function $V$ and the corresponding $\delta$-optimal strategy. We can see in general that, as we proved in Subsection 4.4, the optimal strategy for initial surplus in $D^{1}$ consists in the first branch paying dividends immediately so the surplus moves to $D^{2} \cup \mathcal{M}$. It was proved in Proposition 4.8 that it is not optimal for some initial surplus in $\mathcal{M}$ (unlike the case $c_{2} / c_{1}=b_{2} / b_{1}$ ) to pay dividends so the controlled process remains in the set $\mathcal{M}$ until ruin time. We will see in Figs. 2, 6 and 8 that for some initial surpluses in $\mathcal{M}$ the best local strategy is to get out of $\mathcal{M}$ with the first branch paying dividends immediately. 


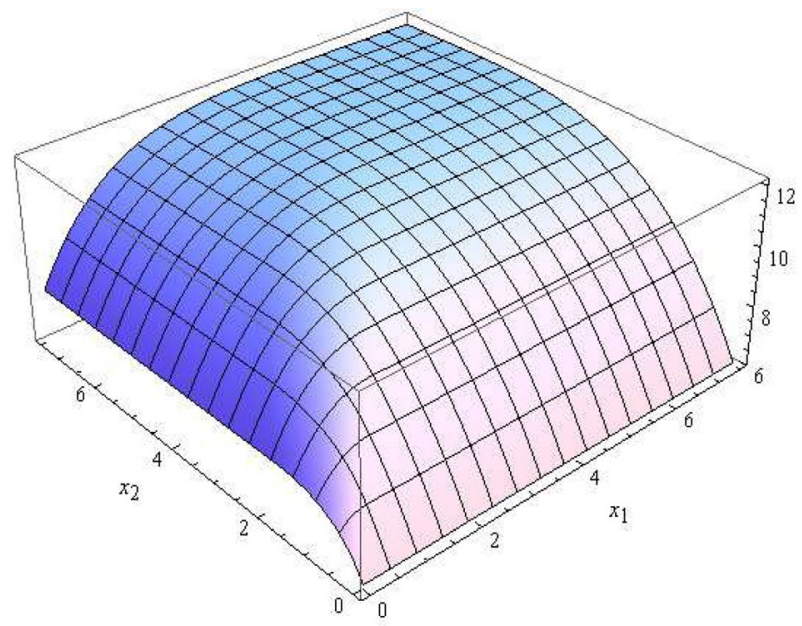

Fig. $3 V^{\delta}$ reduced by $\left(x_{1}+x_{2}\right)$

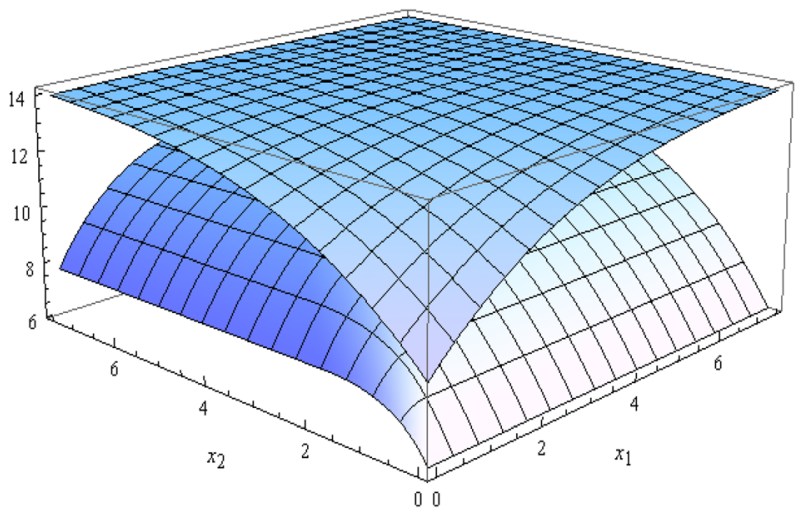

Fig. 4 Comparison $V^{\delta}$ with the value of the merger optimal value function without merger cost

\subsection{Example 1}

We show here a numerical example assuming an exponential claim-size distribution $G(x)=1-e^{-d x}$ with $d=0.6$. In Fig. 2 we show the $\delta$-optimal strategy with $\delta=0.03$. Note that there is a non-action region $\mathcal{C}^{*} \subset D^{2}$. This figure suggests that, as $\delta \rightarrow 0$, the optimal local control in the boundary $\mathcal{A}_{1}^{*}$ should be that the first branch pay some part of the incoming premium as dividends while the second branch pay no dividends, so the bivariate control surplus stays in $\mathcal{A}_{1}^{*}$ and moves rightward to the point $(5.4,6.36) \in \mathcal{A}_{0}^{*}$. By contrast, the optimal strategy in the 


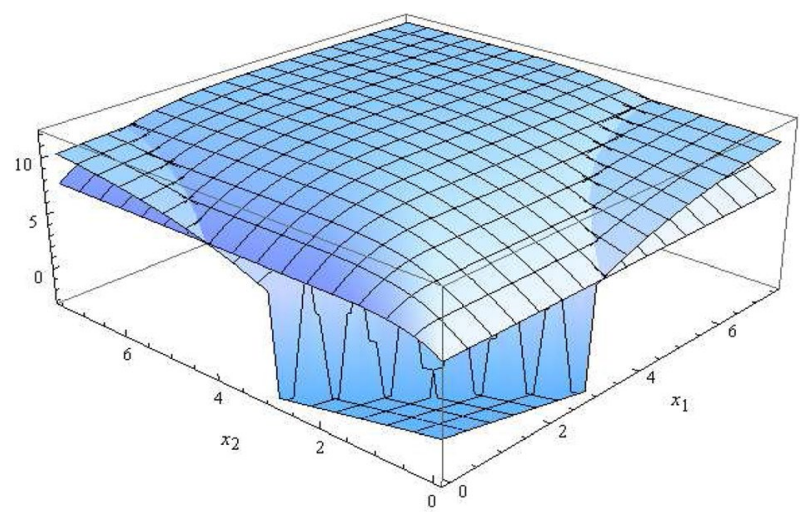

Fig. 5 Comparison $V^{\delta}$ with the value of the merger optimal value function with merger cost $m=3$

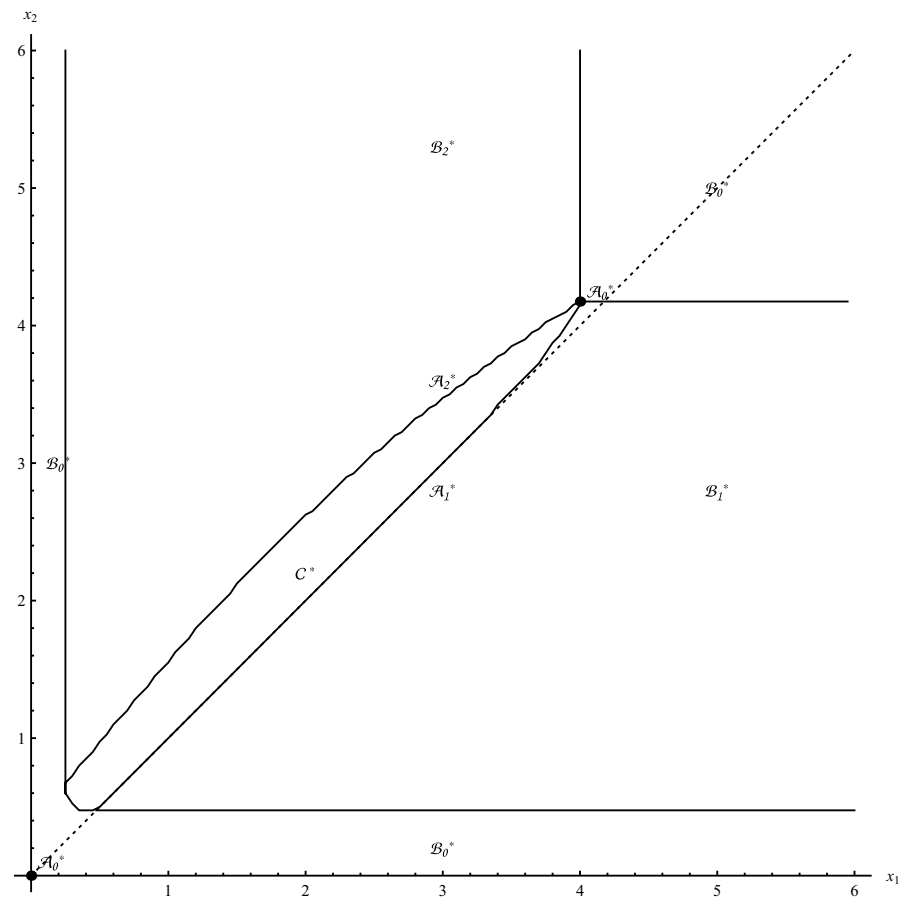

Fig. $6 \delta$-optimal strategy for $\delta=0.025$

boundary $\mathcal{A}_{2}^{*}$ is that none of the two branches pay dividends so the bivariate control surplus moves inside $C^{*}$ because the slope of the boundary $\mathcal{A}_{2}^{*}$ is larger than $c_{2} / c_{1}=1 / 2$. 


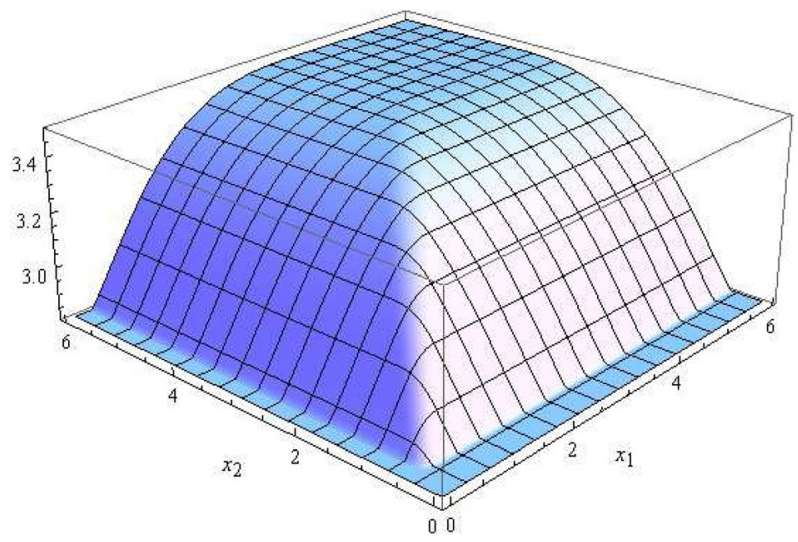

Fig. $7 V^{\delta}$ reduced by $\left(x_{1}+x_{2}\right)$ for $\delta=0.025$

In Fig. 3 we show $V^{\delta}$ reduced by $\left(x_{1}+x_{2}\right)$. As it was noted in Remark 1 , the merger optimal value function without merger cost is greater than $V$. However, this could not be the case when merger costs are considered: we compare $V^{\delta}$ with the value of the merger optimal value function without merger cost in Fig. 4, and with the merger optimal value function with merger cost $m=3$ in Fig. 5. In all the cases the value functions are reduced by $\left(x_{1}+x_{2}\right)$. We see in Fig. 5 that the value function of the merger case with cost only outperforms $V^{\delta}$ when the difference of the surpluses of the two branches is large.

\subsection{Example 2}

We consider here a gamma claim-size distribution

$$
G(x)=1-\left(1+\frac{6}{7} x\right) e^{-\frac{6}{7} x}
$$

We show in Figs. 6 and 7 the $\delta$-optimal strategy and $V^{\delta}$ reduced by $\left(x_{1}+x_{2}\right)$ respectively for $\delta=0.025$. Note that, unlike the previous example, the sets $\mathcal{A}_{0}^{*}=\{(0,0),(4.00,4.75)\}$ and $\mathcal{B}_{0}^{*}$ have two connected components. The graph suggest that $V^{\delta}$ is not differentiable at the lower boundary between $\mathcal{B}_{1}^{*}$ and $\mathcal{B}_{0}^{*}$ but it is differentiable at the upper one (this mirrors the smooth fitting property in the one-dimensional case); a similar observation could be made about the boundaries between $\mathcal{B}_{2}^{*}$ and $\mathcal{B}_{0}^{*}$.

As in the first example, Fig. 6 suggests that the optimal local control in the boundary $\mathcal{A}_{1}^{*}$ should be that the first branch pay some part of the incoming premium as dividends while the second branch pay no dividends, so the bivariate control surplus stays in $\mathcal{A}_{1}^{*}$ and moves rightward to the point $(4.00,4.75) \in \mathcal{A}_{0}^{*}$. Again, the optimal strategy in the boundary $\mathcal{A}_{2}^{*}$ is that none of the two branches pay dividends so the 


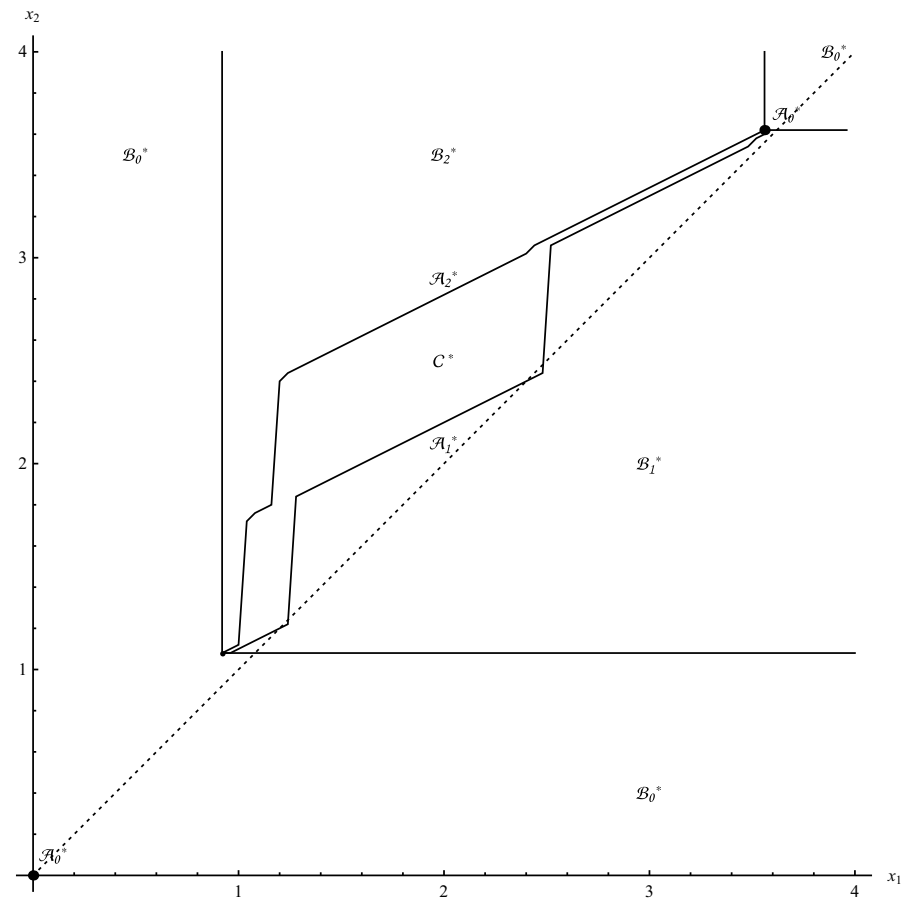

Fig. $8 \delta$-optimal strategy for $\delta=0.02$

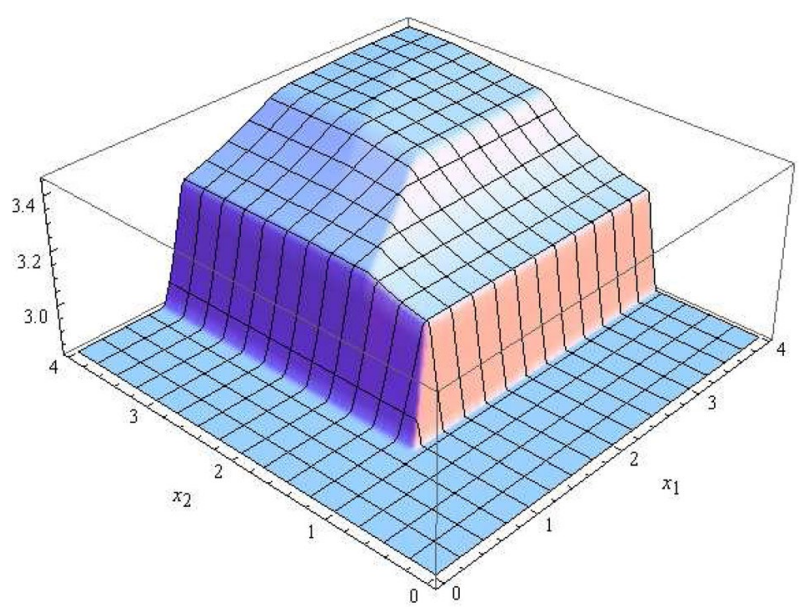

Fig. $9 V^{\delta}$ reduced by $\left(x_{1}+x_{2}\right)$ for $\delta=0.02$ 
bivariate control surplus moves inside $\mathcal{C}^{*}$ because the slope of the boundary $\mathcal{A}_{2}^{*}$ is larger than $c_{2} / c_{1}=1 / 2$.

\subsection{Example 3}

Finally, we consider claims with constant size $\alpha_{0}=29 / 12$. Figs. 8 and 9 show the $\delta$-optimal strategy and $V^{\delta}$ reduced by $\left(x_{1}+x_{2}\right)$ respectively for $\delta=0.02$. As in the previous example, the sets $\mathcal{A}_{0}^{*}=\{(0,0),(3.56,3.62)\}$ and $\mathcal{B}_{0}^{*}$ have two connected components.

Note that there is a relation between the shape of $\mathcal{A}_{1}^{*}$ and $\mathcal{A}_{2}^{*}$, the constant size of the claims $U=29 / 12$ and the rate of growth $c_{2} / c_{1}=1 / 2$ of the uncontrolled bivariate surplus in the event of no claims: $\mathcal{A}_{1}^{*}$ and $\mathcal{A}_{2}^{*}$ contain segments with slope $c_{2} / c_{1}$ and $\Delta x_{1}=b_{1} U$. As in the previous examples, the optimal control strategy in $\mathcal{A}_{2}^{*}$ is that none of the two branches pay dividends, and also the optimal strategy in the boundary $\mathcal{A}_{1}^{*}$ consists on the second branch paying no dividends and the first branch paying some part of the incoming premium as dividends in such a way that the bivariate control surplus stays in $\mathcal{A}_{1}^{*}$ and moves rightward to the point $(3.56,3.62) \in \mathcal{A}_{0}^{*}$. In the segments of $\mathcal{A}_{1}^{*}$ with slope $c_{2} / c_{1}$, the first branch does not need to pay dividends in order to remain in $\mathcal{A}_{1}^{*}$.

\section{Conclusions}

In this paper we have studied the two-dimensional optimal dividend problem in the context of two branches of an insurance company with compound Poisson surplus processes dividing claims and premiums in some specified proportions. We proved that the value function is the smallest viscosity supersolution of the respective Hamilton-Jacobi-Bellman equation and we identified the optimal strategy. In particular, for symmetric case we managed to describe the best strategy explicitly solving some auxiliary equivalent optimization problem. For general case we propose the convergent numerical scheme to find the optimal strategy. Using it, we analyzed some numerical examples.

We believe that this kind of study is still at its first step and several further extension can be done. First of all, one can consider more branches of one company. Moreover, one can think of more general claim arrival process, for example renewal one, with phase-type inter-arrival times. Finally, the analysis may be conducted for the two-dimensional classical risk process perturbed by bivariate correlated Brownian motion. It would be also interesting to consider impulse control and pay-out function related with Gerber-Shiu penalty functions. All of these problems are quite complex and left for future research.

Acknowledgements This research is support by the FP7 Grant PIRSES-GA-2012-318984. Zbigniew Palmowski is supported by the Polish National Science Centre under the Grant 2013/09/B/HS4/01496.

Open Access This article is distributed under the terms of the Creative Commons Attribution 4.0 International License (http://creativecommons.org/licenses/by/4.0/), which permits unrestricted use, distribution, 
and reproduction in any medium, provided you give appropriate credit to the original author(s) and the source, provide a link to the Creative Commons license, and indicate if changes were made.

\section{References}

1. Albrecher H, Azcue P, Muler N (2017) Optimal dividend strategies for two collaborating insurance companies. Adv Appl Probab 49:515-548

2. Albrecher H, Thonhauser S (2009) Optimality results for dividend problems in insurance. Rev R Acad Cienc Exactas Fís Nat Ser A Math RACSAM 103(2):295-320

3. Ambagaspitiya RS (1999) On the distributions of two classes of correlated aggregate claims. Insur Math Econ 24:301-308

4. Avram F, Palmowski Z, Pistorius M (2008) On the optimal dividend problem for a spectrally negative Lévy process. Ann Appl Probab 17(1):156-180

5. Avanzi B (2009) Strategies for dividend distribution: a review. N Am Actuar J 13(2):217-251

6. Avram F, Palmowski Z, Pistorius M (2008) A two-dimensional ruin problem on the positive quadrant. Insur Math Econ 42(1):227-234

7. Avram F, Palmowski Z, Pistorius M (2008) Exit problem of a two-dimensional risk process from the quadrant: exact and asymptotic results. Ann Appl Probab 18(6):2421-2449

8. Azcue P, Muler N (2005) Optimal reinsurance and dividend distribution policies in the CramérLundberg model. Math Financ 15:261-308

9. Azcue P, Muler N (2013) Minimizing the ruin probability allowing investments in two assets: a twodimensional problem. Math Methods Oper Res 77(2):177-206

10. Azcue P, Muler N (2014) Stochastic optimization in insurance: a dynamic programming approach. Springer briefs in quantitative finance. Springer, Berlin

11. Azcue P, Muler N (2018) A multidimensional problem of optimal dividends with irreversible switching: a convergent numerical scheme. arXiv:1804.02547

12. Badescu A, Gong L, Lin S (2015) Optimal capital allocations for a bivariate risk process under a risk sharing strategy. University of Toronto, Toronto (preprint)

13. Badila S, Boxma O, Resing J (2015) Two parallel insurance lines with simultaneous arrivals and risks correlated with inter-arrival times. Insur Math Econ 61:48-61

14. Chan W, Yang H, Zhang L (2003) Some results on the ruin probabilities in a two-dimensional risk model. Insur Math Econ 32:345-358

15. Crandall MG, Lions PL (1983) Viscosity solutions of Hamilton-Jacobi equations. Trans Am Math Soc 277:1-42

16. Czarna I, Palmowski Z (2011) De Finetti's dividend problem and impulse control for a two-dimensional insurance risk process. Stoch Models 27:220-250

17. Denuit M, Genest C, Marceau E (1999) Stochastic bounds on sums of dependent risks. Insur Math Econ 25:85-104

18. Dhaene J, Denuit M (1999) The safest dependence structure among risks. Insur Math Econ 25:11-21

19. Dhaene J, Goovaerts MJ (1996) Dependency of risks and stop-loss order. ASTIN Bull 26:201-212

20. Dhaene J, Goovaerts MJ (1997) On the dependency of risks in the individual life model. Insur Math Econ 19:243-253

21. De Finetti B (1957) Su un'impostazione alternativa dell teoria colletiva del rischio. Trans XV Int Congr Actuar 2:433-443

22. Gerber HU, Shiu ESW (2004) Optimal dividends: analysis with Brownian motion. N Am Actuar J $8: 1-20$

23. Gerber HU (1969) Entscheidungskriterien fuer den zusammengesetzten Poisson-prozess. Schweiz Aktuarver Mitt 1:185-227

24. Gerber HU, Shiu ESW (2006) On the merger of two companies. N Am Actuar J 10:60-67

25. Goovaerts MJ, Dhaene J (1996) The compound Poisson approximation for a portfolio of dependent risks. Insur Math Econ 18:81-85

26. Grandits P, Hubalek F, Schachermayer W, Zigo M (2007) Optimal expected exponential utility of dividend payments in Brownian risk model. Scand Actuar J 2:73-107 
27. Hu T, Wu Z (1999) On the dependence of risks and the stop-loss premiums. Insur Math Econ $24: 323-332$

28. Irbäck J (2003) Asymptotic theory for a risk process with a high dividend barrier. Scand Actuar J 2:97-118

29. Ivanovs J, Boxma O (2015) A bivariate risk model with mutual deficit coverage. Insur Math Econ 64:126-134

30. Jeanblanc M, Shiryaev AN (1995) Optimization of the flow of dividends. Russ Math Surv 50:257-277

31. Kyprianou A, Palmowski Z (2007) Distributional study of De Finetti's dividend problem for a general Lévy insurance risk process. J Appl Probab 44(2):428-443

32. Loeffen R (2008) On optimality of the barrier strategy in de Finetti's dividend problem for spectrally negative Lévy processes. Ann Appl Probab 18(5):1669-1680

33. Loeffen RL, Renaud J-F (2010) De Finetti's optimal dividends problem with an affine penalty function at ruin. Insur Math Econ 46(1):98-108

34. Müller A (1997) Stop-loss order for portfolios of dependent risks. Insur Math Econ 21:219-223

35. Müller A (1997) Stochastic orderings generated by integrals: a unified study. Adv Appl Probab 29:414-428

36. Schmidli H (2008) Stochastic control in insurance. Probability and its applications. Springer, London

37. Soner HM (1988) Optimal control of jump-Markov processes and viscosity solutions. In: Fleming W, Lions PL (eds) Stochastic differential systems, stochastic control theory and applications. IMA volumes in mathematics and its applications, vol 10, Springer-Verlag, New York, pp 501-511

38. Thonhauser S, Albrecher H (2007) Dividend maximization under consideration of the time value of ruin. Insur Math Econ 41:163-184

39. Sundt B (1999) On multivariate Panjer recursions. ASTIN Bull 29(1):29-45

40. Zajic T (2000) Optimal dividend payout under compound Poisson income. J Optim Theory Appl 104:195-213

41. Zhou X (2005) On a classical risk model with a constant dividend barrier. N Am Actuar J 9:1-14 